\title{
Effect of electron-phonon interaction on the formation of one-dimensional electronic states in coupled $\mathrm{Cl}$ vacancies
}

\author{
Bruno Schuler, ${ }^{1,{ }^{*}}$ Mats Persson, ${ }^{2,3}$ Sami Paavilainen, ${ }^{4}$ Niko Pavliček, ${ }^{1}$ Leo Gross, ${ }^{1}$ Gerhard Meyer, ${ }^{1}$ and Jascha Repp ${ }^{5}$ \\ ${ }^{1}$ IBM Research-Zurich, Säumerstrasse 4, 8803 Rüschlikon, Switzerland \\ ${ }^{2}$ Surface Science Research Centre, Department of Chemistry, University of Liverpool, Liverpool, L69 3BX, United Kingdom \\ ${ }^{3}$ Department of Applied Physics, Chalmers University of Technology, SE 41296, Göteborg, Sweden \\ ${ }^{4}$ Department of Physics, Tampere University of Technology, 33720 Tampere, Finland \\ ${ }^{5}$ Institute of Experimental and Applied Physics, University of Regensburg, 93053 Regensburg, Germany \\ (Received 30 March 2015; revised manuscript received 16 May 2015; published 24 June 2015)
}

\begin{abstract}
The formation of extended electron states in one-dimensional nanostructures is of key importance for the function of molecular electronic devices. Here, we study the effects of strong electron-phonon interaction on the formation of extended electronic states in intentionally created $\mathrm{Cl}$ vacancy pairs and chains in a $\mathrm{NaCl}$ bilayer on $\mathrm{Cu}(111)$. The interaction between the vacancies was tailored by fabricating vacancy pairs and chains of different orientation and separation with atomic precision using vertical manipulation. Small vacancy separations led to the formation of quantum-well-like vacancy states and localized interface states. By using scanning tunneling spectroscopy, we measured their energy splitting and broadening as a function of the intervacancy separation. Remarkably, the energy splitting between the vacancy states is enlarged by level repulsion resulting from the phonon dressing of the electronic states, as evidenced by theory.
\end{abstract}

DOI: 10.1103/PhysRevB.91.235443

PACS number(s): 68.37.Ef, 68.55.Ln, 73.20.-r, 63.20.kd

\section{INTRODUCTION}

One-dimensional nanostructures assembled from single metal atoms and molecules on surfaces are of great fundamental and technological interest as their electronic states exhibit a wealth of new quantum phenomena that might be exploited in future molecular electronics devices [1]. Some examples of such phenomena identified by scanning tunneling microscopy (STM) and spectroscopy (STS) include metal-atom chains exhibiting "particle-in-box" states [2], bistable antiferromagnetism [3], Tomonaga-Luttinger liquid behavior [4], and signs of Majorana spinors [5]. The formation of extended or bandlike electronic states in metal-atom and molecular chains on surfaces has been widely studied experimentally [6-10], but potentially interesting effects of strong electron-phonon coupling on these states, such as, for instance, polaronic effects, have attracted much less attention. A notable exception is the observation of coherent electron-phonon states and the breakdown of the Born-Oppenheimer approximation in oligothiophene molecular chains [11]. In the electronically adiabatic regime, where the Franck-Condon principle is applicable, the electron-phonon coupling results in vibronic satellite structures. How these vibronic effects influence the delocalization of an injected electron in a one-dimensional nanostructure is an open question, in particular when the electron-phonon coupling is so strong that these effects dominate.

Here, we address this question in a combined experimental and theoretical study of coupled electronic states localized at intentionally created $\mathrm{Cl}$ divacancies and vacancy chains in a $\mathrm{NaCl}$ bilayer on $\mathrm{Cu}(111)$. The $\mathrm{Cl}$ vacancies provide a quantumwell structure that could be created with atomic precision using the STM tip [12,13]. The energies of the electronic states formed in these structures by injection of an electron from the tip of an STM and their spatial behavior on the atomic scale

\footnotetext{
*bsc@zurich.ibm.com
}

were resolved by STS and analyzed using simple models. Our findings have ramifications for the formation of extended states from coupled adsorbate or confined defect states (such as in dopants [14] or dangling bonds [15]) in semiconductors and ionic crystals. Hence it could improve the understanding of polaronic effects in coupled quantum systems, such as quantum dots [16-21], charge-based qubits [22,23], and quantum cellular automata [24].

Coupled, individual $\mathrm{Cl}$ vacancies in a $\mathrm{NaCl}$ bilayer on a $\mathrm{Cu}(111)$ surface are ideal model systems to study the effect of strong electron-phonon coupling on the delocalization of electronic states. First, $\mathrm{Cl}$ monovacancies are atomically well-defined and highly symmetric defects. They feature two distinct electronic states: an unoccupied vacancy state (VS) that is strongly coupled to optical phonons in the film and a localized interface-state (LIS) with a negligible electronphonon coupling $[25,26]$. The electronic coupling between VSs or LISs of different vacancies can be tuned in a controlled way by the lateral spacing between vacancies with atomic precision [27]. Finally, $\mathrm{Cl}$ vacancies feature a much higher stability against inelastic excitations than adsorbates [28]. $\mathrm{Cl}$ vacancies could even be filled with other atoms [29]. Hence they allow us to explore artificial coupled quantum systems with great control.

\section{EXPERIMENT}

The experiments were carried out in a home-built lowtemperature combined STM and atomic force microscopy (AFM) operated at $5 \mathrm{~K}$. A $\mathrm{Cu}(111)$ single-crystal sample was cleaned by several sputtering and annealing cycles. $\mathrm{NaCl}$ was evaporated thermally, keeping the sample temperature at about $270 \mathrm{~K}$, such that defect-free, (100)-terminated $\mathrm{NaCl}$ bilayer islands were formed [30,31]. Bias voltages refer to the sample voltage with respect to the tip. In the spectroscopic measurements, the tunneling conductance $d I / d V$ was recorded with conventional lock-in techniques with an ac 

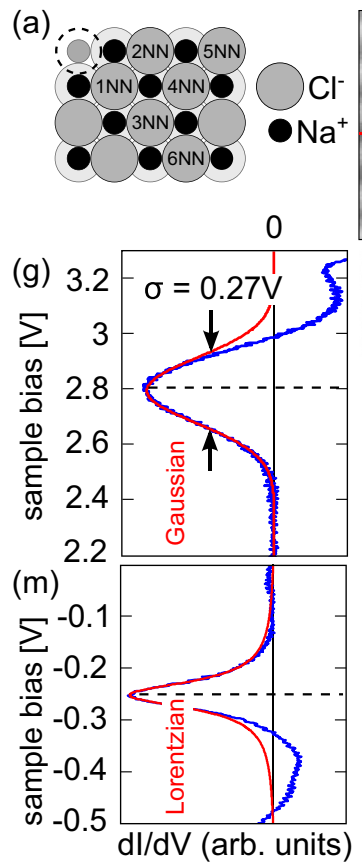
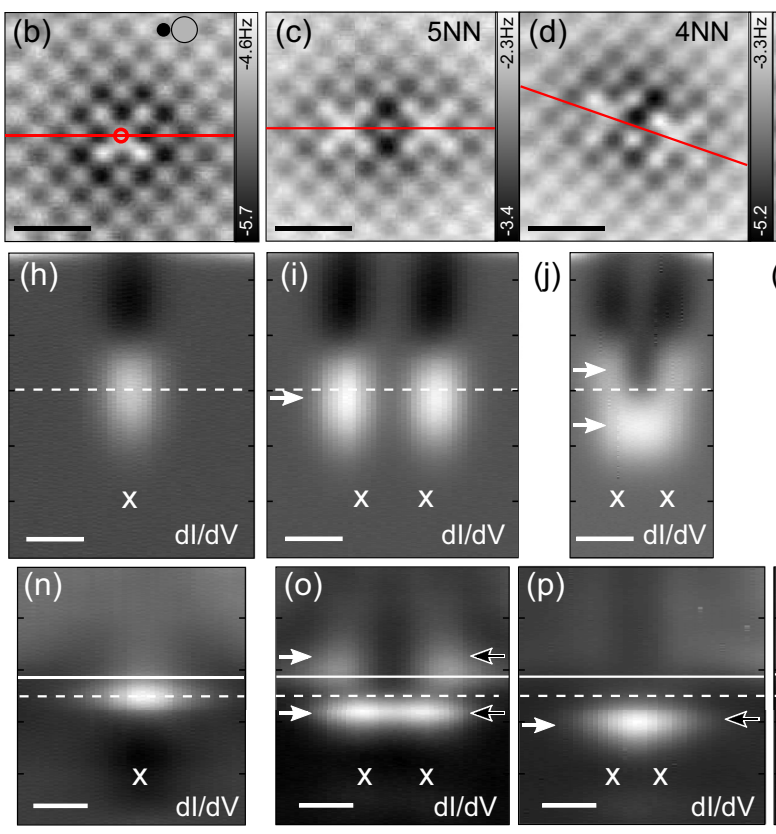
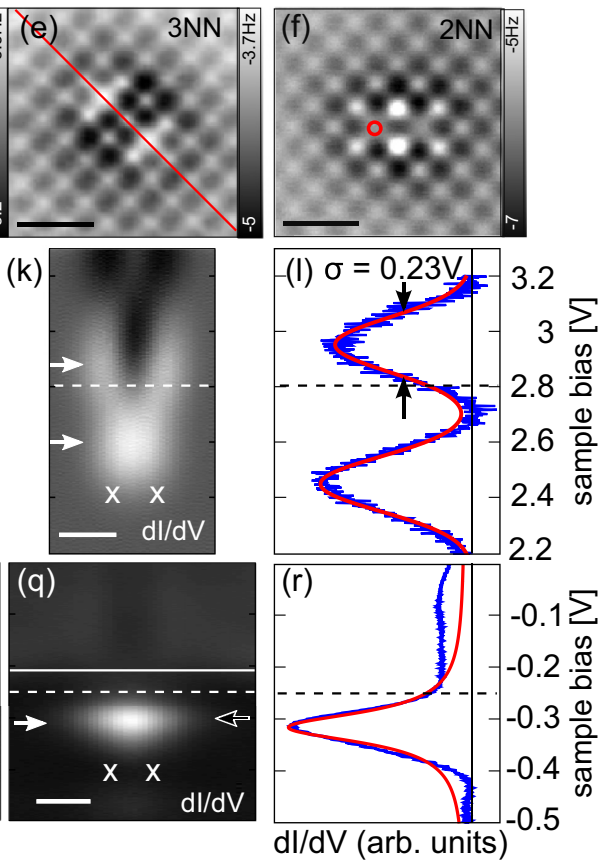

FIG. 1. (Color online) (a) $\mathrm{NaCl}$ bilayer model with a $\mathrm{Cl}$ vacancy (dashed circle) and its nearest $\mathrm{Cl}$ neighbors indicated. (b)-(f) CO tip AFM images of a single $\mathrm{Cl}$ vacancy (b) and divacancies in the $5 \mathrm{NN}(\mathrm{c}), 4 \mathrm{NN}(\mathrm{d}), 3 \mathrm{NN}(\mathrm{e})$, and $2 \mathrm{NN}$ (f) configurations. (g) and (l) $d I / d V$ spectra of the monovacancy and 2NN divacancy VS peaks with a Gaussian fit in red, recorded at the red circles in (b) and (f). (h)-(k) $d I / d V(x, V)$ maps along the red lines in (b)-(e) [same $V$ scale as in (g)]. Peaks in $d I / d V$ are displayed as bright, whereas the darkest areas mark regions of negative differential conductance (NDC). ${ }^{1}$ The dashed line indicates the monovacancy VS peak position. (m) and (r) $d I / d V$ spectra of the LIS of a monovacancy and a $2 \mathrm{NN}$ divacancy with a Lorentzian fit in red, recorded at the red circles in (b) and (f). (n)-(q) $d I / d V(x, V)$ maps along the red lines indicated in (b)-(e) [same $V$ scale as in (m)]. The dashed line indicates the monovacancy LIS peak position and the solid line the interface-state band onset. White crosses indicate the vacancy positions. White arrows mark the peak positions of the observed $d I / d V$ resonances. Black arrows in (n)-(q) mark the peak positions of the calculated LDOS using the $\sigma$-wave multiple scattering model. Scale bar is $10 \AA$.

bias amplitude of $25 \mathrm{mV}$ at a frequency of $294 \mathrm{~Hz}$. In the double-barrier tunneling junction geometry, the voltage drop across the insulating film will cause a voltage-dependent shift of the electronic levels. This tip-induced Stark shift is a few percent of the applied bias but depends only weakly on the tip distance. Therefore it is not considered here [32]. The vacancies were created by bringing the tip into a controlled contact with the $\mathrm{NaCl}$ surface by approaching a sharp $\mathrm{Cu}$ tip by about 4.5-5.5 $\AA$ at zero bias from an STM set-point of $(I=2 \mathrm{pA}$ and $V=0.2 \mathrm{~V})$. Thereby a $\mathrm{Cl}$ atom was transferred to the tip apex, as evidenced by a characteristic contrast change in STM images and a remaining depression at the predefined $\mathrm{Cl}$ site. The resulting $\mathrm{Cl}$ at the tip was removed and a $\mathrm{Cu}$ tip was reestablished by indenting into the $\mathrm{Cu}$ surface. $\mathrm{A} \mathrm{Cl}$ vacancy was usually created with more than $90 \%$ probability and with about $90 \%$ probability at the atomically predefined location. Crucial factor was the sharpness of the $\mathrm{Cu}$ tip, which was assessed by the STM corrugation of CO molecules on the surface. Atomic resolution images taken before the vacancy creation ensured that the vacancies will be created precisely at the desired location. Constant-height AFM images with a $\mathrm{CO}$ tip $[33,34]$ were recorded to identify the vacancy location and exclude the generation of other close-by defects. In addition, we measured the local contact potential difference on the polar film with Kelvin probe force microscopy to ensure that the defects are indeed $\mathrm{Cl}$ vacancies [35].
In a previous study [32], single $\mathrm{Cl}$ vacancies in a $\mathrm{NaCl}$ bilayer on copper surfaces were characterized in detail. $\mathrm{A} \mathrm{Cl}$ vacancy in the outermost layer of this supported $\mathrm{NaCl}$ bilayer is analogous to the widely studied and well-known color centers in bulk $\mathrm{NaCl}$ [36] with one key difference: the localized VS is unoccupied because the electron can tunnel into unoccupied metal states. The unoccupied VS then gives rise to a positively charged vacancy and an attractive potential that is able to split off a LIS from the free-electron-like interface-state band of the $\mathrm{NaCl} / \mathrm{Cu}(111)$ [37]. This LIS was observed as a narrow resonance just below the bottom of the interface-state band in the $d I / d V$ spectra, whereas the VS showed up as a broad negative ion resonance at a sample voltage of about $2.8 \mathrm{~V}$ in $d I / d V$. The negative ion resonance was found to have a large Gaussian broadening (full width half maximum (FWHM) of $\sigma=0.27 \mathrm{~V}$ ) because of the strong electron-phonon coupling of the electron in the VS to optical phonons in the $\mathrm{NaCl}$ bilayer. Despite this strong coupling corresponding to an estimated Huang-Rhys parameter of about $S=30$, the associated relaxation energy of the electron in the VS is not large enough to prohibit tunneling into unoccupied metal states and to allow

\footnotetext{
${ }^{1}$ At positive voltages, the NDC arises from the increase of the tunnel barrier for resonant tunneling through the VS with voltages greater than the VS [26].
} 


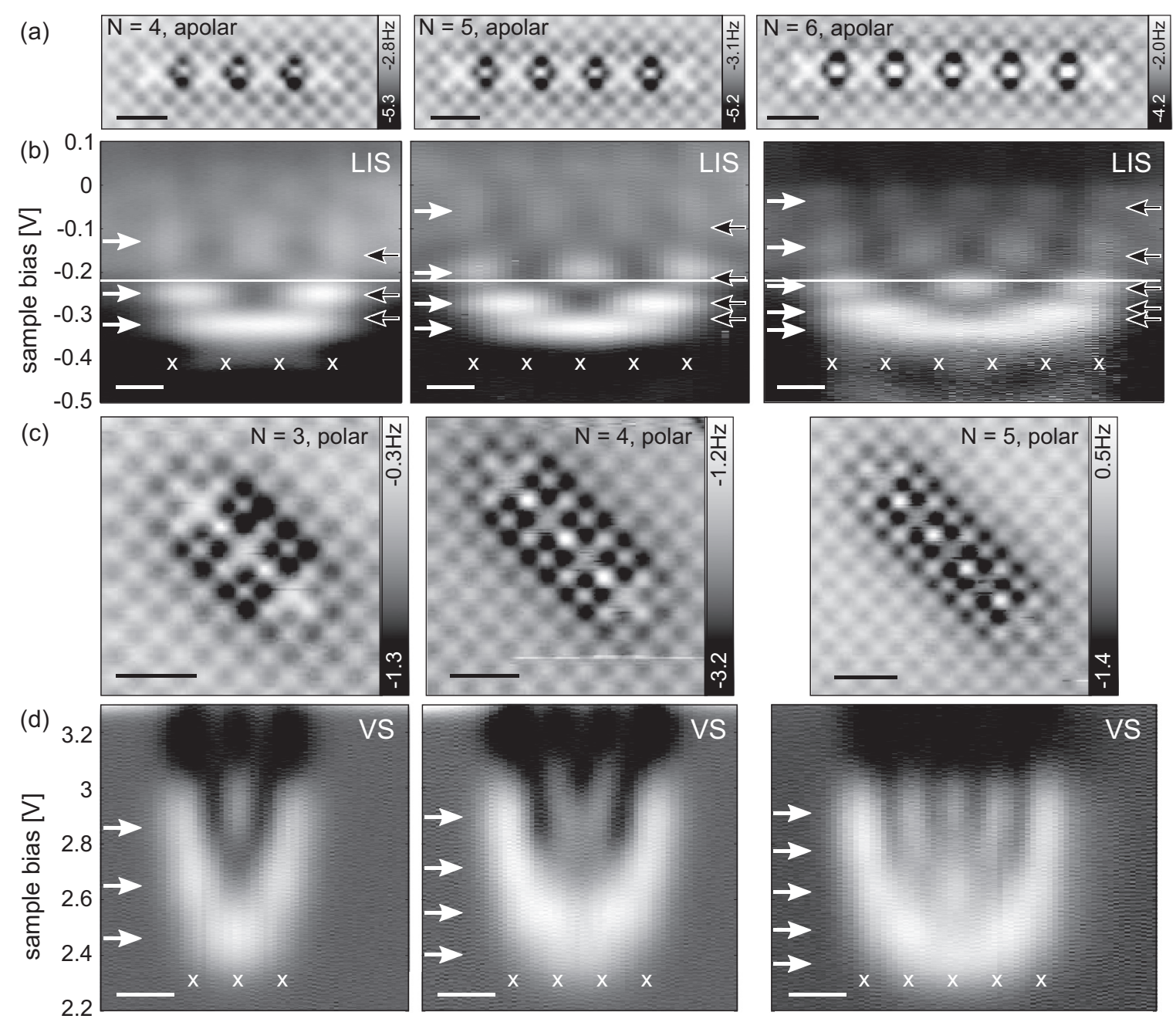

FIG. 2. (a) $\mathrm{CO}$ tip $\mathrm{AFM}$ images of $\mathrm{Cl}$ vacancy chains with $N=4,5,6$ vacancies in the $5 \mathrm{NN}$ configuration (apolar direction). (b) Corresponding $d I / d V(x, V)$ maps of the LISs along the chains. The continuous line indicates the interface-state band onset. White arrows mark the peak positions of the observed $d I / d V$ resonances, and black arrows mark the peak positions of the calculated LDOS. (c) CO tip AFM images of $\mathrm{Cl}$ vacancy chains with $N=3,4,5$ vacancies in the $3 \mathrm{NN}$ configuration (polar direction). (d) Corresponding $d I / d V(x, V)$ maps of the VSs along the chains. White crosses mark the positions of the vacancies and the white arrows $d I / d V$ peaks. Scale bars: $10 \AA$.

the formation of a stable occupied VS corresponding to a localized polaron. In contrast to the VS, the LIS was found to be dominated by lifetime broadening with a negligible phonon broadening in the $d I / d V$ spectra, owing to the efficient screening of the electron-phonon interaction by the metal electrons.

First, vacancy pairs were created with different separations. Divacancies in the ninth-nearest-neighbor (9NN), 6NN, 5NN, $4 \mathrm{NN}, 3 \mathrm{NN}$, and $2 \mathrm{NN}^{2}$ configurations [see schematic in Fig. 1(a)] were created from two $\mathrm{Cl}$ vacancies close to each other but not in the vicinity of any other vacancies or defects. Constant-height AFM images of the $5 \mathrm{NN}$ to $2 \mathrm{NN}$ divacancies and of a single vacancy are shown in Figs. 1(b)-1(f). Using a CO tip, the vacancies were recognized as a bright crosslike feature in AFM maps. The electronic structure of the divacan-

\footnotetext{
${ }^{2}$ The $2 \mathrm{NN}$ divacancy was less stable than the divacancies with larger separations.
}

cies was characterized by single $d I / d V$ spectra or constantheight $d I / d V(x, V)$ maps along the line connecting the two vacancies. The VS resonances are shown in Figs. 1(g)-1(1) and the LIS resonances in Figs. 1(m)-1(r), respectively. Note that all $d I / d V$ data shown here were recorded using $\mathrm{Cu}$-terminated tips. For divacancies with a separation larger than $10 \AA$ (i.e., larger than a 4NN divacancy), a single VS resonance was observed to be localized at each vacancy site separately with an energy similar to the one of the monovacancy. For smaller separations (4NN-2NN divacancies), however, two peaks with Gaussian line shapes appeared around $2.8 \mathrm{~V}$. The $d I / d V(x, V)$ maps show that the state at lower energy is localized between the vacancies (symmetric), whereas the state at higher energy has a nodal plane between them (antisymmetric), in analogy with the bonding and antibonding orbitals of the hydrogen molecule.

Similarly, the divacancy LISs were also observed to split into a symmetric and an antisymmetric state. However, for 
(a)

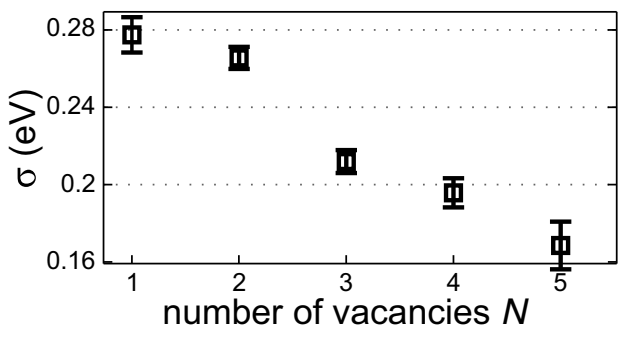

(b) tight-binding (TB)

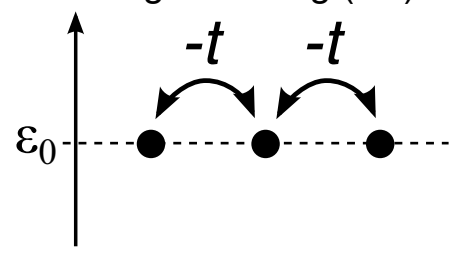

(c) TB + on-site energy shift

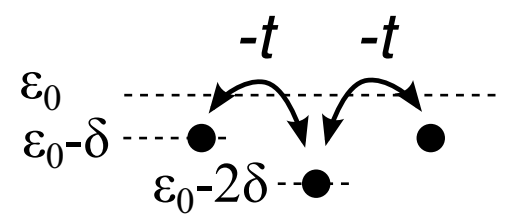

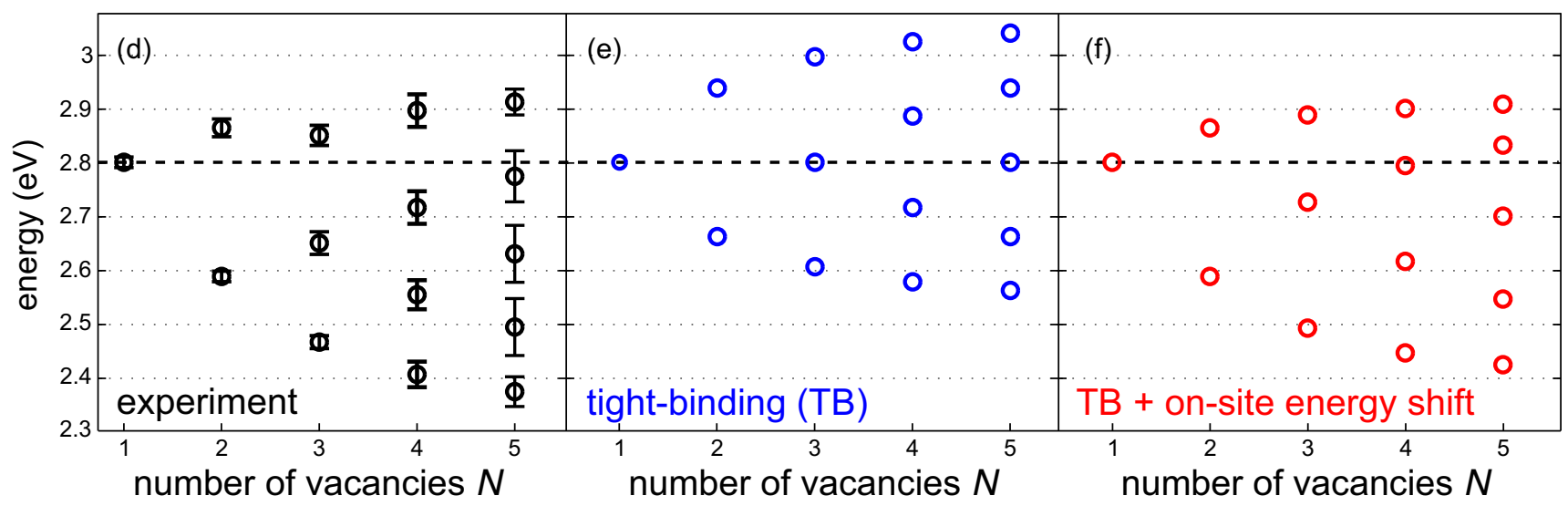

FIG. 3. (Color online) (a) Experimental VS broadening $\sigma$ as a function of the $3 \mathrm{NN}$ chain length. (b) Tight-binding (TB) model for a $N=3$ chain. (c) TB model including the electrostatic on-site energy shift between nearest neighbors. (d) Experimental VS energies as a function of the $3 \mathrm{NN}$ chain length. (e) TB model energies obtained by fitting the on-site energy $\varepsilon_{0}=2.8 \mathrm{eV}$ and hopping term $t=0.14 \mathrm{eV}$ to the experimental mono- and divacancy VS energies shown in (d). (f) TB model accounting for a shift of the on-site energy $(\delta=0.07 \mathrm{eV})$ due to the electrostatic interaction between nearest neighbors.

small vacancy separations, the latter state lies above the $\mathrm{NaCl} / \mathrm{Cu}(111)$ interface band onset at $\varepsilon_{B}=-230 \mathrm{mV}$ and overlaps with the band continuum. Hence only the symmetric state is observed as a well-defined peak in $d I / d V$. The splitting of both the VSs and LISs was found to increase with decreasing inter-vacancy distance. The VSs did not split symmetrically about the monovacancy energy but their mean value was shifted towards lower energies. In addition, the VSs showed a tendency to have a smaller level broadening with decreasing separation.

To study the delocalization of VSs and LISs further, short one-dimensional arrays of $N$ vacancies, referred to in the following as vacancy chains, were created in the $5 \mathrm{NN}$ (apolar [001] $\mathrm{NaCl}$ direction) and the $3 \mathrm{NN}$ (polar [011] $\mathrm{NaCl}$ direction) configuration. They were also characterized by constant-height CO tip AFM images [Figs. 2(a) and 2(c)]. For the $5 \mathrm{NN}$ chains, the LISs form one-dimensional quantum-well states, observed as distinct resonances in $d I / d V$, with an increasing integer number of nodal planes with voltage [see Figs. 2(b) and 5]. Remarkably, also broader resonances are observed up to $200-300 \mathrm{mV}$ above the interface-state band onset. These quasibound LISs have a lower height in $d I / d V$, owing to the increased lifetime broadening compared to the bound LISs. This originates from the additional decay channel into the interface-state band. Furthermore, the energy position of the ground-state LIS does not change significantly from the $N=4$ to the $N=6$ chain and seems to converge to about $-0.33 \mathrm{~V}$. The VSs of the $5 \mathrm{NN}$ chains only show a single resonance peak at about $2.7 \mathrm{~V}$, localized at each vacancy site because of the weak electronic coupling. In contrast, the 3NN chains displayed in Fig. 2(c) are subject to significant interaction between the VSs and exhibit several resonances, as shown in Fig. 2(d). The increasing number of nodal planes with voltage shows that the VSs are delocalized over the entire chains despite the strong electron-phonon coupling. Notably, the level broadening decreases for longer chains as displayed in Fig. 3(a). This effect is a direct evidence of the dependence between the electron-phonon coupling and the enhanced delocalization for longer chains. It can be rationalized by the larger spatial extent of the eigenstates, which decreases their relaxation energy and accordingly the effective electron-phonon coupling.

\section{THEORY AND DISCUSSION}

In the following, the VS energies of the $3 \mathrm{NN}$ chains are compared with the results from a simple tight-binding (TB) model. First, we assume that each vacancy in the chain has an on-site energy $\varepsilon_{0}$ and is coupled to its next neighbor(s) with a hopping term $-t$, as sketched in Fig. 3(b). By adjusting the free fitting parameters $\varepsilon_{0}$ and $t$ to the experimental mono- and divacancy VS energies, one can calculate the energy levels for different chain lengths as shown in Fig. 3(e). In comparison with the experimental energy spectrum, depicted in Fig. 3(d), the level spacings for each chain could be roughly reproduced, but the TB model does not capture the shift of the band center towards lower energies with increasing chain length (as already described for the divacancies). This shift can be explained by 
the electrostatic interaction between adjacent vacancies that deepens the potential at each vacancy and therefore lowers the on-site energy. To account for the energy shift, we introduce the parameter $\delta$, which is the on-site energy shift due to a neighboring vacancy [see Fig. 3(c)]. The resulting energy levels of this extended TB model are shown in Fig. 3(f). The calculated energy spectrum now agrees very well with the experimentally observed VS levels of the chains.

So far, the strong electron-phonon coupling, as shown by the large broadening of the VSs, was neglected in our tight-binding models. The role of this coupling in the formation of delocalized states by a tunneling electron in vacancy pairs (and chains) needs to be investigated. Here, we use a simple generalization of the electron-phonon interaction model of the single vacancy [32] to vacancy pairs and chains. As detailed in Appendix C2, the direct electronic interactions between the VSs are described by a simple tight-binding model, and each VS is coupled linearly to the phonon modes of the $\mathrm{NaCl}$ film. As we will show next, in this model, the strong electron-phonon coupling has a profound effect on the energy splitting and broadening of the vacancy states. This effect is illustrated here for a divacancy using appropriate parameters, where the electronic interaction between the vacancies is large compared to the characteristic phonon energies, corresponding to an electronically adiabatic regime.

In Figs. 4(a) and 4(b), sections of the adiabatic potential energy surfaces (PES) of the singly occupied, divacancy states are shown, as obtained from a constrained minimization with respect to the phonon normal coordinates (ionic positions) for fixed values of on-site energy fluctuations $\delta \varepsilon_{1}$ and $\delta \varepsilon_{2}$ of vacancy 1 and 2 , respectively (see Appendix C2). The sections of the PESs corresponding to symmetric vacancy energy fluctuations $\delta \epsilon_{s}$ (where $\delta \epsilon_{1}=\delta \epsilon_{2}$ ) [Fig. 4(a)] are split by $2 t$, where $-t$ is the direct electronic interaction between the VSs, and both have the same shape as for the adiabatic PES of a monovacancy but with half the relaxation energy at the minimum. In contrast, for antisymmetric vacancy energy fluctuations $\delta \epsilon_{a s}$ (where $\delta \epsilon_{1}=-\delta \epsilon_{2}$ ) [Fig. 4(b)], the corresponding sections of the PESs exhibit an avoided crossing with a minimum energy gap of $2 t$. This avoided crossing will be shown to have a profound influence on the phonon broadening and the formation of delocalized states.

As for the single vacancy, the result for the phonon broadening of the tunneling through the divacancy states turns out to have a simple physical form in the prevailing strong electron-phonon coupling and electronically adiabatic limits, as detailed in Appendix C 2. The phonon broadening is then determined by probabilities for vertical Franck-Condon transitions from the vibrational ground-state of the PES of the electronic ground-state to vibrational states on the two PESs for the divacancy states [gray arrows in Figs. 4(c) and 4(d)]. In the strong electron-phonon coupling limit, the transition probabilities are dominated by the contribution from the linear electron-phonon coupling terms. The corresponding phonon broadenings are then simply obtained from the changes in the divacancy energy levels $\tilde{\varepsilon}_{b}$ (bonding state) and $\tilde{\varepsilon}_{a}$ (antibonding state) with the Gaussian fluctuations of the energies $\delta \varepsilon_{1}$ and $\delta \varepsilon_{2}$ due to the zero-point motions of the phonons.

The strong coupling result for the phonon broadening of the divacancy states is illustrated in Figs. 4(c) and 4(d). A symmetric fluctuation $\delta \varepsilon_{s}$ (where $\delta \varepsilon_{1}=\delta \varepsilon_{2}$ ) results, as shown in Fig. 4(c), in a linear dependence of $\tilde{\varepsilon}_{b}$ and $\tilde{\varepsilon}_{a}$ on $\delta \varepsilon_{s}$ with a slope of one, but they are split by $2 t$. These Gaussian fluctuations then give rise to two overlapping Gaussian line shapes centered at $\varepsilon_{0} \pm t$ with a variance of $\left\langle\delta \varepsilon_{s}^{2}\right\rangle$ for the local density of states (LDOS).

In contrast, the level repulsion results in a minimum energy separation $2 t$ between $\tilde{\varepsilon}_{b}$ and $\tilde{\varepsilon}_{a}$ for an antisymmetric fluctuation $\delta \varepsilon_{a s}$ (where $\delta \varepsilon_{1}=-\delta \varepsilon_{2}$ ). Furthermore, $\delta \varepsilon_{a s}$ breaks the symmetric and antisymmetric character of the two coupled $\mathrm{VSs}$, and they tend to localize on each vacancy for $\delta \varepsilon_{a s} \gg t$. The resulting LDOS from these Gaussian fluctuations, shown in Fig. 4(d), consists of two relatively sharp, highly asymmetric line shapes, with mean energies separated by more than the energy splitting $2 t$. In general, the fluctuations $\delta \varepsilon_{1}$ and $\delta \varepsilon_{2}$ of the two vacancy energies will be a superposition of both $\delta \varepsilon_{s}$ and $\delta \varepsilon_{a s}$. In the case of uncorrelated vacancy energy fluctuations $\left\langle\delta \varepsilon_{1} \delta \varepsilon_{2}\right\rangle=0$, the LDOS is a convolution of the line shape from $\delta \varepsilon_{a s}$ with a Gaussian line shape with a variance of $\left\langle\delta \varepsilon_{1,2}^{2}\right\rangle / 2$ because $\delta \varepsilon_{s}$ and $\delta \varepsilon_{a s}$ contribute equally to $\left\langle\delta \varepsilon_{1,2}^{2}\right\rangle$, the variance of the single vacancy energy fluctuations. As the Gaussian shape dominates the broadening in such a convolution, the resulting LDOS as displayed in Fig. 4(e) has a similar shape as two Gaussians with a FWHM reduced by almost $1 / \sqrt{2}$ as compared to an isolated vacancy. This decrease of the broadening in the uncorrelated case is the underlying reason why the observed broadenings of the levels of the chains decrease with chain size.

For instance, uncorrelated vacancy site energy fluctuations can arise in situations where the vacancy energy fluctuations are dominated by localized modes at each vacancy such as optical phonons with nearly flat dispersions. In reality, however, a given individual phonon mode may act on the energy of both vacancies in a symmetric or antisymmetric fashion (consider e.g. the motion of an ion centered between two vacancies). Hence partially correlated vacancy site energy fluctuations cannot be excluded. Our experimental results are consistent with the assumption of fully uncorrelated fluctuations of the two VSs. Interestingly, in the uncorrelated case, the LDOS, shown in Fig. 4(e) (red line), exhibits two peaks with an apparent separation that is substantially larger than the intrinsic, purely electronic splitting of $2 t$. This result is a characteristic effect of the level repulsion between $\tilde{\varepsilon}_{b}$ and $\tilde{\varepsilon}_{a}$ and is in sharp contrast to a superposition of two monovacancy resonances separated by the intrinsic splitting [shown in Fig. 4(e) (blue line)]. In the latter case, one would not be able to identify separate peaks due to the larger broadening and smaller splitting.

The enhancement of the apparent peak separation in the uncorrelated case over the intrinsic splitting is shown in Fig. 4(f) as a function of the scaled intrinsic energy splitting $2 t / \sigma$, where $\sigma$ is the broadening of a single VS. We define the apparent peak separation $\Delta \tilde{\varepsilon}$ as the separation between the centers of the two Gaussian line shapes with broadening (FWHM) $\tilde{\sigma}$ fitted to the calculated LDOS. The relative enhancement $\Delta \tilde{\varepsilon} / 2 t$ of the apparent splitting is most important for small $t$. For large $t$, the splitting of divacancy energy levels approaches the intrinsic energy splitting of $2 t$. As for the single vacancy level, the broadening of a divacancy level 

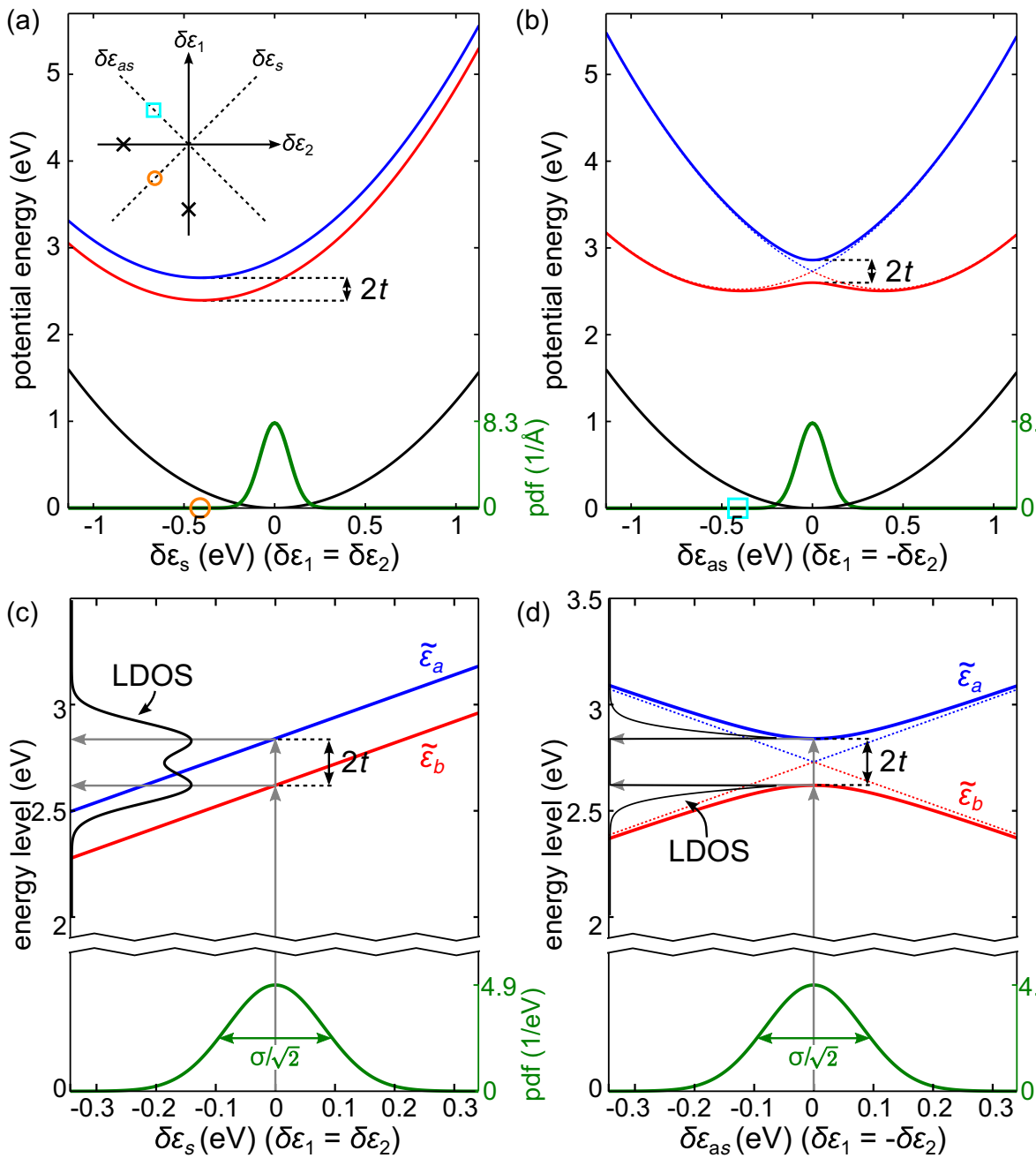

(e)

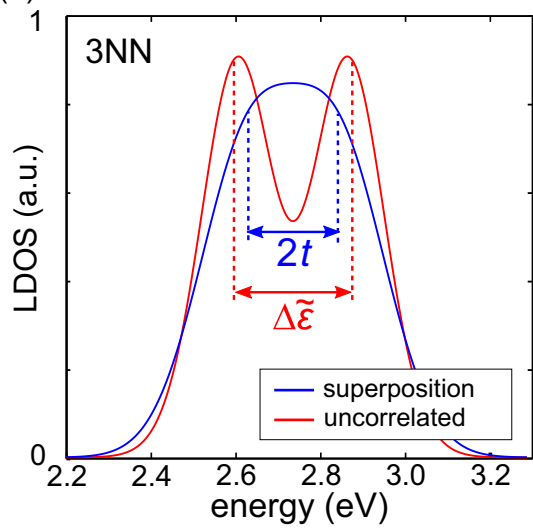

(d)

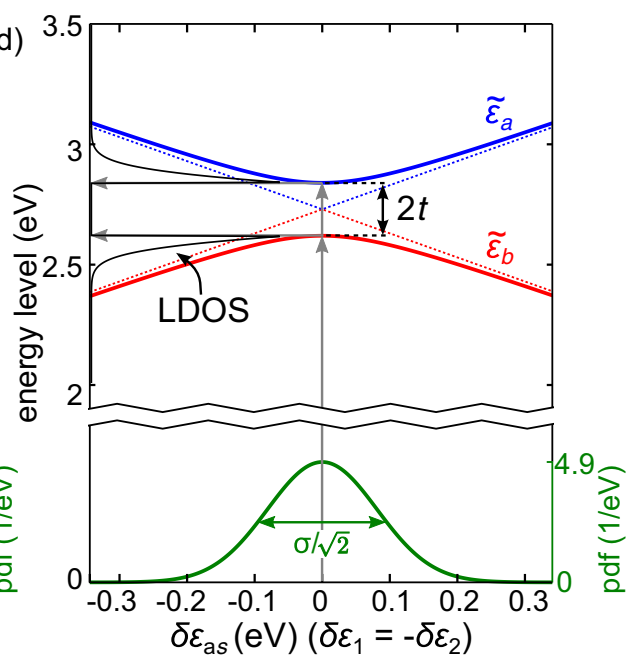

(f)

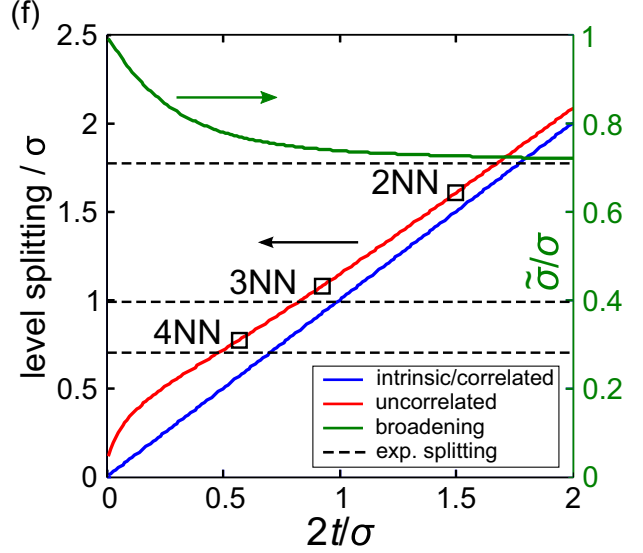

FIG. 4. (Color online) (a) and (b) Calculated adiabatic potential energy surfaces (PESs) of the electronic ground-state (black) and the electronically excited and singly occupied divacancy states (red and blue) as a function of symmetric and antisymmetric on-site vacancy energy fluctuations $\delta \varepsilon_{s}$ (a) and $\delta \varepsilon_{a s}$ (b), respectively (see Appendix $\mathrm{C} 2$ ). The vibrational groundstate probability distribution functions (pdf) of $\delta \varepsilon_{s}$ (a) and $\delta \varepsilon_{a s}$ (b) are indicated by the green curves. Here, $2 t$ is the intrinsic electronic level splitting. The inset in (a) shows $\delta \varepsilon_{s}$ and $\delta \varepsilon_{a s}$ in terms of the on-site vacancy energy fluctuations $\delta \varepsilon_{1}$ and $\delta \varepsilon_{2}$. The black crosses mark the equilibrium values of $\delta \varepsilon_{1}$ and $\delta \varepsilon_{2}$ of the two energy minima when the divacancy is occupied by a single electron. (c) and (d) Energy-level diagram for the divacancy states as a function of $\delta \varepsilon_{s}$ (c) and $\delta \varepsilon_{a s}$ (d). The vertical gray arrows indicate the electron-attachment process and the black curves the resulting LDOS. (e) Calculated LDOS of the $3 \mathrm{NN}$ divacancy for uncorrelated vacancy energy fluctuations (red), which is compared to a superposition of two Gaussians separated by $2 t$ and each with a broadening $\sigma$ (blue). $\Delta \tilde{\varepsilon}$ denotes the level splitting for uncorrelated vacancy energy fluctuations. (f) Scaled level splitting $\Delta \tilde{\varepsilon} / \sigma$ as obtained from a Gaussian fit to the calculated line shapes of the divacancy states for uncorrelated vacancy energy fluctuations (red) compared to the intrinsic splitting (blue) as a function of the scaled intrinsic level splitting $2 t / \sigma$. The calculated and observed scaled splittings for different divacancy configurations are indicated by black squares and dashed horizontal lines, respectively. The green curve shows the apparent level broadening $\tilde{\sigma}$ for uncorrelated vacancy energy fluctuations. then scales with the square root of its relaxation energy (see Appendix C 2).

The hopping terms $t$ can be extracted from Fig. 4(f) by taking $\Delta \tilde{\varepsilon}$ equal to the observed splitting and using the single vacancy value $0.27 \mathrm{eV}$ for $\sigma$. For example, $t=0.11 \mathrm{eV}$ was obtained from $\Delta \tilde{\varepsilon}=0.28 \mathrm{eV}$ for the $3 \mathrm{NN}$ divacancy. The values obtained for the hopping terms $t$ are very reasonable as supported by density-functional theory (DFT) calculations of the unoccupied Kohn-Sham states of $\mathrm{Cl}$ divacancies in $\mathrm{NaCl}(2 \mathrm{ML}) / \mathrm{Cu}(111)$. As shown in Fig. 4(f), the calculated values for $2 t$ of the various divacancy configurations are close to the values for the intrinsic splittings extracted from experimental data. However, the absolute divacancy energies are underestimated by the DFT calculations, as expected [see Fig. 6(e)].

The coupling between the LISs of $\mathrm{Cl}$ divacancies and chains were studied in a simple, multiple $\sigma$-wave (zero angular momentum in 2D) scattering model of the interfacestate scattering from the positively charged vacancies (see Appendix C 3). The results from this model, as indicated in Figs. 1(o)-1(q), are able to fully capture the observed behavior of the coupled LISs. The absence of a LIS doublet below the 
onset of the interface-state band is simply understood by the relatively strong interaction between the weakly bound LISs of the two vacancies at small intervacancy distances. With increasing distances between the vacancies, this interaction decreases, and a second LIS appears below the band onset. The observed symmetric character of the LIS below the band edge and antisymmetric character of the extended LIS above $\varepsilon_{B}$ for the $3 \mathrm{NN}$ vacancy are also revealed in the calculated LDOS images (see Fig. 8). Furthermore, the obtained energies for the LISs of the vacancy chains are in excellent agreement with the experiments, as indicated in Fig. 2(b).

\section{CONCLUSIONS}

In summary, from an AFM and STS study, we find that the localized VSs and LISs at intentionally created $\mathrm{Cl}$ divacancies in a $\mathrm{NaCl}$ bilayer on $\mathrm{Cu}(111)$ form symmetric and antisymmetric states, in analogy to the bonding and antibonding orbitals of a hydrogen molecule. As expected, the energy splitting between these states increases with decreasing intervacancy distance. A comparison with theory shows that the energy splitting of the VSs is significantly enlarged by the strong coupling of the tunneling electrons with phonons in the $\mathrm{NaCl}$ film. Furthermore, VSs and LISs of vacancy chains form one-dimensional quantum-well states. The VS levels of the chains could be well described by a simple tight-binding model that takes the electrostatic interaction between neighboring vacancies into account. The model provides also a microscopic understanding why the level broadening is reduced with increasing chain size.

\section{ACKNOWLEDGMENTS}

We thank R. Allenspach for comments and acknowledge financial support from the ERC Advanced Grant CEMAS (agreement no. 291194) and the EU projects PAMS (610446) and QTea (317485). Allocation of computer resources through SNAC and CSC is gratefully acknowledged.

\section{APPENDIX A: DISTANCE DEPENDENCE OF THE VSS AND LISS OF DIFFERENT CL DIVACANCIES}

Table I lists all measured and calculated values of the VS and LIS for different divacancy geometries. The calculations are explained in the subsequent paragraphs. For the VSs, the experimental energies were obtained by fitting two Gaussians to the $d I / d V(V)$ spectra. The theoretical results were obtained from a phonon-broadening model, in which the intrinsic electronic splitting parameter $t$ was calculated by densityfunctional theory (DFT) (see Appendix C for details).

In the case of the LISs, the experimental energies were obtained by fitting two Lorentzians to the $d I / d V(V)$ spectra. The theoretical results were obtained from a phenomenological scattering model with parameters taken from independent experiments (see Appendix C 3 for details). For both VSs and LISs, the experimental and theoretical results demonstrate increasing level splitting with decreasing intervacancy distance.

\section{APPENDIX B: CL VACANCY CHAINS $d I / d V(x, y)$ MAPS}

In Fig. 5, constant-height AFM and $d I / d V(x, y)$ maps of $\mathrm{Cl}$ vacancy chains $(N=4,5,6)$ in the $5 \mathrm{NN}$ configuration are shown. Each $d I / d V(x, y)$ map is recorded at the bias corresponding to a LIS resonance. The states have the typical shape of $1 \mathrm{D}$ quantum-well eigenstates. Above the $\mathrm{NaCl} / \mathrm{Cu}(111)$ interface band onset at $\varepsilon_{B}=-230 \mathrm{mV}$, however, these states are obscured by the states of the $2 \mathrm{D}$ free-electron band at the interface that give rise to standing-wave patterns [31].

\section{APPENDIX C: MODELING OF VACANCY STATES AND LOCALIZED INTERFACE-STATES}

The tunneling through the divacancy states in the absence of electron-phonon coupling has been simulated by DFT calculations, whereas the phonon broadening of the VSs of divacancies and longer chains has been modeled by a generalization of our earlier phonon-broadening model for a single vacancy in the strong electron-vibration coupling limit [32]. In the case of the LIS of mono- and divacancies,

TABLE I. Distance dependence of VS and LIS of Cl divacancies. Experimental and theoretical energy positions of the VSs and LISs [obtained from a superposition of two Gaussians (for VSs) or Lorentzians (for LISs)] of the $d I / d V$ measurements and LDOS for Cl divacancies at various intervacancy distances $d . E_{\mathrm{s}}$ and $E_{\text {as }}$ refer to the observed energy positions of the symmetric and antisymmetric states, and $\Delta E$ denotes the corresponding level splitting. For the VSs, $\Delta \varepsilon$ is the level splitting obtained in the phonon broadening model using the level splittings $2 t$ obtained from the DFT calculations, whereas for the LISs, $\Delta \varepsilon$ is the energy difference between the energies $\varepsilon_{\mathrm{s}}$ and $\varepsilon_{\text {as }}$ of the symmetric and antisymmetric states in the $\sigma$-wave, multiple scattering model.

\begin{tabular}{|c|c|c|c|c|c|c|c|c|c|c|c|c|}
\hline & \multirow[b]{2}{*}{$d(\AA)$} & \multicolumn{3}{|c|}{ VS (experiment) } & \multicolumn{2}{|c|}{ VS (theory) } & \multicolumn{3}{|c|}{ LIS (experiment) } & \multicolumn{3}{|c|}{ LIS (theory) } \\
\hline & & $E_{\mathrm{s}}(\mathrm{V})$ & $E_{\text {as }}(\mathrm{V})$ & $\Delta E(\mathrm{~V})$ & $2 t$ & $\Delta \varepsilon(\mathrm{eV})$ & $E_{\mathrm{s}}(\mathrm{V})$ & $E_{\mathrm{as}}(\mathrm{V})$ & $\Delta E(\mathrm{~V})$ & $\varepsilon_{\mathrm{s}}(\mathrm{eV})$ & $\varepsilon_{\mathrm{as}}(\mathrm{eV})$ & $\Delta \varepsilon(\mathrm{eV})$ \\
\hline $2 \mathrm{NN}$ & 5.6 & 2.43 & 2.92 & 0.49 & 0.42 & 0.45 & -0.315 & $\mathrm{n} / \mathrm{a}$ & $\mathrm{n} / \mathrm{a}$ & -0.322 & $\mathrm{n} / \mathrm{a}$ & $\mathrm{n} / \mathrm{a}$ \\
\hline $3 \mathrm{NN}$ & 7.8 & 2.58 & 2.86 & 0.28 & 0.26 & 0.30 & -0.308 & $\mathrm{n} / \mathrm{a}$ & $\mathrm{n} / \mathrm{a}$ & -0.299 & $\mathrm{n} / \mathrm{a}$ & $\mathrm{n} / \mathrm{a}$ \\
\hline $4 \mathrm{NN}$ & 8.7 & 2.67 & 2.85 & 0.18 & 0.16 & 0.21 & -0.304 & $\mathrm{n} / \mathrm{a}$ & $\mathrm{n} / \mathrm{a}$ & -0.293 & $\mathrm{n} / \mathrm{a}$ & $\mathrm{n} / \mathrm{a}$ \\
\hline $5 \mathrm{NN}$ & 11.3 & 2.77 & $\mathrm{n} / \mathrm{a}$ & $\mathrm{n} / \mathrm{a}$ & & & -0.282 & -0.169 & 0.113 & -0.281 & -0.168 & 0.113 \\
\hline $6 \mathrm{NN}$ & 12 & 2.73 & 2.75 & 0.02 & & & -0.29 & -0.133 & 0.157 & -0.279 & -0.177 & 0.102 \\
\hline $9 \mathrm{NN}$ & 16 & 2.74 & $\mathrm{n} / \mathrm{a}$ & $\mathrm{n} / \mathrm{a}$ & & & -0.277 & -0.158 & 0.119 & -0.269 & -0.210 & 0.059 \\
\hline single & $\infty$ & 2.8 & $\mathrm{n} / \mathrm{a}$ & $\mathrm{n} / \mathrm{a}$ & & & -0.249 & $\mathrm{n} / \mathrm{a}$ & $\mathrm{n} / \mathrm{a}$ & $-0.249^{\mathrm{a}}$ & $\mathrm{n} / \mathrm{a}$ & $\mathrm{n} / \mathrm{a}$ \\
\hline
\end{tabular}

${ }^{a}$ Model parameter taken from measurements. 


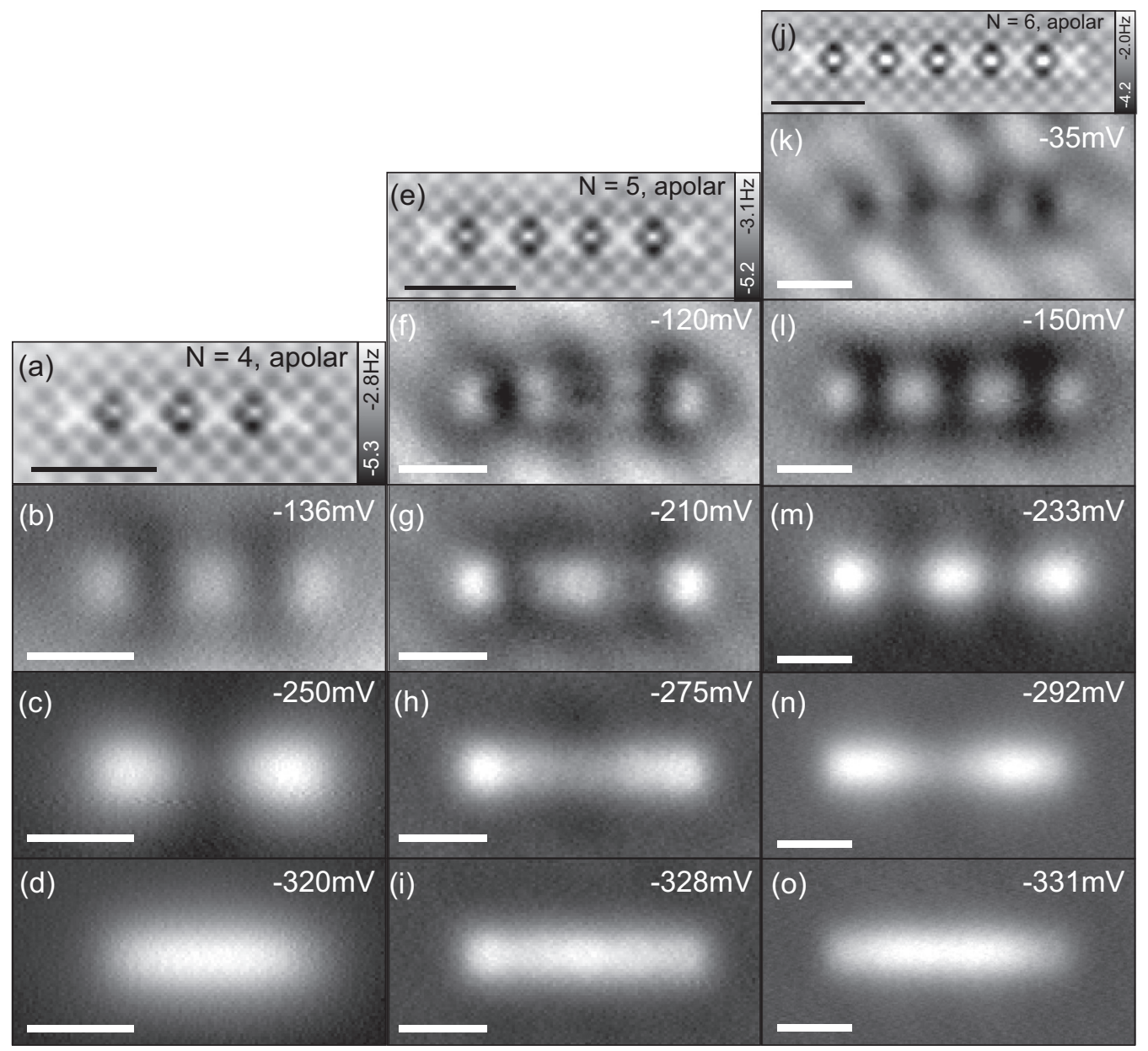

FIG. 5. (a), (e), and (j) Constant-height AFM images of the 5NN chain with $N=4,5$, or $6 \mathrm{Cl}$ vacancies with a CO tip. (b)-(d) Constantheight $d I / d V(x, y)$ maps of the LISs of the $N=4$ chain. (f)-(i) Constant-height $d I / d V(x, y)$ maps of the LISs of the $N=5$ chain. (k)-(o) Constant-height $d I / d V(x, y)$ maps of the LISs of the $N=6$ chain. Note that the standing-wave patterns [31] above the interface-state band onset $\left(\varepsilon_{B}=-230 \mathrm{mV}\right)$ partly conceal the quantum-well states. Scale bars: $20 \AA$.

a DFT treatment is not practical because of the large system size required to describe the interface-state band, and we used instead a simple phenomenological model for the multiple scattering of the interface-state electrons from the vacancies. The parameters of this model were determined by the observed interface state dispersion and bound state energy of the LIS for a single vacancy.

In all cases, the modeling of electron tunneling through the chain of vacancies is based on the Tersoff-Hamann (TH) approximation [38,39]. This approximation for the tunneling amounts to a simplified description of the tip using a $\sigma$-wave approximation for the electrons emitted from the tip apex and a Bardeen approximation for the tunneling, which is justified at large tip-surface distances and low biases $V$. The differential tunneling current $\frac{d I}{d V}(V)$ then has a simple and transparent form in terms of the local density of states as

$$
\frac{d I}{d V}(V) \propto-\frac{1}{\pi} \operatorname{Im}\left\langle\vec{r}_{t}\left|\hat{g}\left(\varepsilon_{F}+e V\right)\right| \vec{r}_{t}\right\rangle,
$$

where $\vec{r}_{t}$ is the position of the tip apex and $\varepsilon_{F}$ is the Fermi energy. In principle, $\hat{g}(\varepsilon)$ is the one-electron Green function for the interacting electron system, including electron-phonon coupling.

\section{DFT calculations of divacancy states}

The divacancies $2 \mathrm{NN}, 3 \mathrm{NN}$, $4 \mathrm{NN}$, and $5 \mathrm{NN}^{3}$ were geometrically optimized in the DFT calculations (see main text for details of the calculations). The resulting relaxed structures and the corresponding changes in atomic positions are illustrated in Figs. 6(c) and 6(d) for the 3NN and 5NN divacancies. These changes are similar to those obtained for a monovacancy, as shown in Figs. 6(a) and 6(b).

The calculated LDOS images at the energies of the VS peak doublet in the calculated spectra, shown in the insets of Fig. 6(e), corroborate the experimental finding that the VS at lower energy is symmetric and the state at the higher energy is antisymmetric. The calculated energies of the symmetric

\footnotetext{
${ }^{3}$ Note that because of periodic boundary conditions, the $5 \mathrm{NN}$ divacancy in the supercell chosen corresponds to an infinite chain of vacancies.
} 

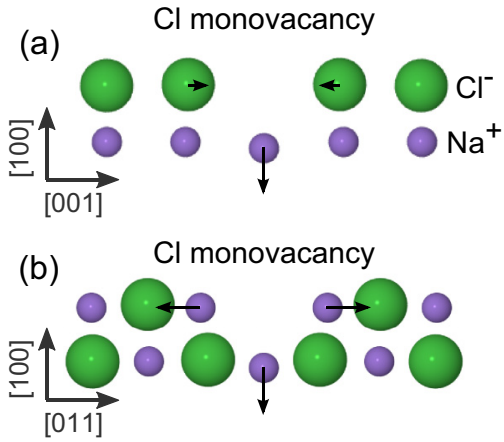
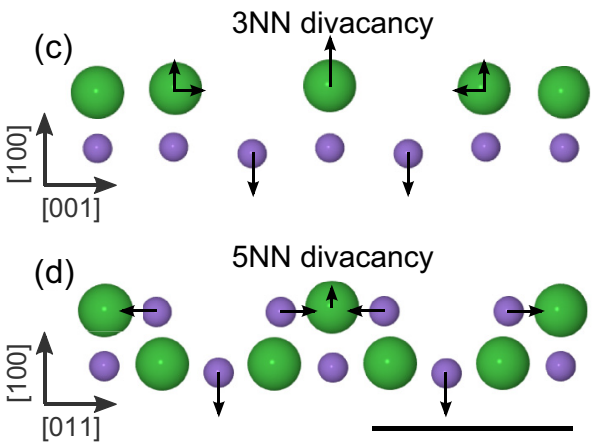

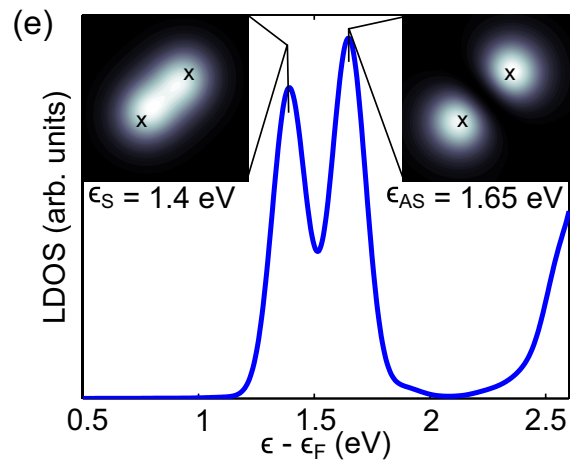

FIG. 6. (Color online) (a) and (b) $\mathrm{Cl}$ monovacancy geometry in the polar (a) and apolar (b) direction. Largest displacements around a vacancy: nearest $\mathrm{Na}$ : $0.28 \AA$ (lateral), Na below vacancy: $-0.34 \AA$ (vertical). For clarity, only the $\mathrm{NaCl}$ bilayer is shown. (c) and (d) $\mathrm{Cl}$ divacancy in the $3 \mathrm{NN}$ (c) and $5 \mathrm{NN}$ (d) configurations. Largest displacements around a $3 \mathrm{NN}$ divacancy: nearest Cl: $0.13 / 0.23 \AA$ (lateral/vertical), middle Cl: $0.38 \AA$ (vertical), nearest Na: $0.29 \AA$ (lateral), Na below vacancy: $-0.32 \AA$ (vertical). Largest displacements around a $5 \mathrm{NN}$ divacancy: nearest Cl: $0.15 / 0.22 \AA$ (lateral/vertical), middle Cl: $0.16 \AA$ (vertical), nearest Na: $0.27 \AA$ (lateral), Na below vacancy: $-0.32 \AA$ (vertical). Black arrows indicate the relative strength of the relaxations of ions that are displaced by more than $0.1 \AA$. (e) Calculated LDOS at $6 \AA$ above a vacancy site of the $3 \mathrm{NN}$ divacancy in the $\mathrm{NaCl}$ bilayer on $\mathrm{Cu}(111)$. The insets show LDOS maps at $6 \AA$ above the uppermost NaCl plane at the energies corresponding to the symmetric and antisymmetric VS. Scale bar is $10 \AA$.

and antisymmetric $3 \mathrm{NN}$ VS correspond to $\varepsilon_{s}=1.4 \mathrm{eV}$ and $\varepsilon_{a s}=1.65 \mathrm{eV}$, respectively. Note that the calculated energies correspond to one-electron energies of unoccupied KohnSham states and not to the excited state energies corresponding to the electron attachment energies to the resonance states. As previously found in the case of the monovacancy [32], the Kohn-Sham energy underestimates this attachment energy by roughly $0.5-1 \mathrm{eV}$. However, the relative energies of unoccupied states are typically much better described by DFT calculations. The values obtained for the energy difference between the symmetric and antisymmetric states for the $2 \mathrm{NN}, 3 \mathrm{NN}$, and $4 \mathrm{NN}$ are shown in Table I. These values are used as input parameters in the phonon-broadening model described below, as they are only due to the electronic coupling between the states.

\section{Phonon broadening of chain vacancy states}

In our previous theoretical treatment of the resonance tunneling through a single VS, we applied a simple resonancelevel model coupled to a single Einstein phonon mode [32]. In particular, we showed that the strong electron-phonon coupling limit, which prevails in our case, results in a Gaussian envelope of the VS line shape in the resonance tunneling spectra. Here we generalize this model to resonant tunneling through the states of a linear chain of vacancies (including a divacancy) but now coupled to a general phonon bath characterised implicitly by a correlation function for the phonon fluctuations of the on-site vacancy energies. In particular, we consider the two extreme limits for this function corresponding to uncorrelated and fully correlated phonon fluctuations of the on-site vacancy energies. Furthermore, as in the case of the single VS, we solve this model in the strong electron-phonon coupling limit. To ease the presentation, the formalism is developed at zero temperature but the final results for the LDOS are also valid at nonzero temperature.

The electronic part of the single resonance level Hamiltonian is straightforwardly generalised to $N$ resonance levels as

$$
\hat{H}_{\mathrm{e}}^{(0)}=\hat{H}_{\mathrm{a}}^{(0)}+\hat{H}_{\mathrm{am}},
$$

where

$$
\hat{H}_{\mathrm{a}}^{(0)}=\varepsilon_{a}^{(0)} \sum_{i}\left|\psi_{a i}\right\rangle\left\langle\psi_{a i}\right|-\sum_{\langle i, j\rangle} t\left(\left|\psi_{a i}\right\rangle\left\langle\psi_{a j}\right|+\text { H.c. }\right),
$$

is the Hamiltonian for the vacancy states and

$$
\hat{H}_{\mathrm{am}}=\sum_{k} \varepsilon_{k}\left|\psi_{k}\right\rangle\left\langle\psi_{k}\right|+\sum_{i} \sum_{k}\left(V_{i k}\left|\psi_{a i}\right\rangle\left\langle\psi_{k}\right|+\text { H.c. }\right) .
$$

is the Hamiltonian describing the interaction of the vacancy states with the metal states. Here $\left\langle\vec{r} \mid \psi_{a i}\right\rangle$ are wave functions of the multiple VSs, labeled by $i(i=1,2, \ldots, N)$, with degenerate energies $\varepsilon_{a}^{(0)}$, and $-t<0$ is the direct interaction energy between nearest-neighboring $(\langle i, j\rangle)$ VSs. The eigenstates of $\hat{H}_{\mathrm{a}}^{(0)}$ turn into resonances through the interaction $V_{i k}$ with the band of metal states $\left|\psi_{k}\right\rangle$ with energies $\varepsilon_{k}$. The phonon part of the Hamiltonian has the general form

$$
\hat{H}_{\mathrm{v}}=\sum_{\lambda}\left(\frac{p_{\lambda}^{2}}{2}+\frac{\omega_{\lambda}^{2} q_{\lambda}^{2}}{2}\right)
$$

Here, $q_{\lambda}$ is the normal coordinate of the phonon mode $\lambda$ with energy $\hbar \omega_{\lambda}$ and $p_{\lambda}$ is the associated momentum. The phonons are assumed to couple linearly in $q_{\lambda}$ to the on-site energy of the VS at $i$. Thus the electron-phonon coupling $\hat{H}_{\mathrm{ev}}$ is only through the on-site energies and is then, as in the Holstein Hamiltonian, given by

$$
\hat{H}_{\mathrm{ev}}=\sum_{\lambda} \varepsilon_{a i \lambda}^{\prime} q_{\lambda}\left|\psi_{a i}\right\rangle\left\langle\psi_{a i}\right|
$$

Here the coupling strength $\varepsilon_{a i \lambda}^{\prime}$ is given by the first derivative of the vacancy energy $\varepsilon_{a i}$ of site $i$ with respect to the normal coordinate $q_{\lambda}$.

To proceed, we need the local density of states of the electron at the position of the tip apex $\vec{r}_{t}$, which according 
to Eq. $(\mathrm{C} 1)$ is given by

$$
\rho\left(\varepsilon, \vec{r}_{t}\right)=-\frac{1}{\pi} \operatorname{Im}\left(\sum_{i, j}\left\langle\vec{r}_{t} \mid \psi_{a i}\right\rangle g_{a i j}(\varepsilon)\left\langle\psi_{a j} \mid \vec{r}_{t}\right\rangle\right),
$$

where the matrix elements of the Green function with respect to the impurity states are given by

$$
g_{a i j}(\varepsilon)=\left\langle\psi_{a i}\right|\left\langle\left\{0_{\lambda}\right\}_{\lambda}\left|\left(\varepsilon+i 0^{+}-\hat{H}\right)^{-1}\right|\left\{0_{\lambda}\right\}_{\lambda} \mid \psi_{a j}\right\rangle .
$$

Here, $\left|\left\{0_{\lambda}\right\}_{\lambda}\right\rangle=\prod_{\lambda}\left|0_{\lambda}\right\rangle$ is the ground state for the phonon system and $\left|0_{\lambda}\right\rangle$ is the ground state of the phonon $\lambda$ and $\hat{H}=$ $\hat{H}_{\mathrm{e}}^{(0)}+\hat{H}_{\mathrm{v}}+\hat{H}_{\mathrm{ev}}$. Eliminating the metal states in the wide metal band limit, one obtains in a standard manner:

$$
\begin{aligned}
g_{a i j}(\varepsilon)= & \left\langle\left\{0_{\lambda}\right\}_{\lambda}\right|\left\langle\psi_{a i}\right|\left[(\varepsilon+i \gamma) \hat{I}_{a}-\hat{H}_{\mathrm{a}}^{(0)}-\hat{H}_{\mathrm{ev}}-\hat{H}_{\mathrm{v}}\right]^{-1} \\
& \times\left|\psi_{a j}\right\rangle\left|\left\{0_{\lambda}\right\}_{\lambda}\right\rangle,
\end{aligned}
$$

where $\gamma$ is the energy-independent resonance broadening of the VSs due to their coupling with the metal states. Here, we have neglected the indirect couplings between the VSs mediated by the metal states, which are expected to be negligible for the weak coupling of the VSs to the metal states. A further key simplification arises in the prevailing strong electron-vibration coupling and narrow resonance limits, in which $\hat{H}_{\mathrm{v}}$ can be neglected compared to $\hat{H}_{\mathrm{ev}}$ and a negligible life time broadening $\gamma$ compared to the phonon broadening of the electronic states,

$$
\begin{aligned}
\rho_{a i j}(\varepsilon) \equiv & -\frac{1}{2 \pi i}\left(g_{a i j}(\varepsilon)-g_{a j i}^{*}(\varepsilon)\right) \\
\approx & \int_{\ldots} \int_{\lambda} d q_{\lambda}\left|\left\langle q_{\lambda} \mid 0_{\lambda}\right\rangle\right|^{2}\left\langle\psi_{a i}\right| \\
& \times \delta\left(\varepsilon \hat{I}_{a}-\tilde{H}_{\mathrm{a}}^{(0)}-\tilde{H}_{\mathrm{ev}}\right)\left|\psi_{a j}\right\rangle,
\end{aligned}
$$

where

$$
\left\langle q \mid 0_{\lambda}\right\rangle=\frac{1}{\sqrt{\delta q_{\lambda} \sqrt{2 \pi}}} \exp \left[-\left(\frac{q}{2 \delta q_{\lambda}}\right)^{2}\right]
$$

is the ground-state wave function of the phonon mode $\lambda$. The zero-point fluctuations of the phonon normal coordinates $q_{\lambda}$ associated with these wave functions result in a multivariate Gaussian distribution of fluctuations of the vacancy on-site energies:

$$
\delta \varepsilon_{i}=\varepsilon_{a i}-\varepsilon_{a}^{(0)}=\sum_{\lambda} \varepsilon_{a i \lambda}^{\prime} q_{\lambda}
$$

The multidimensional integral over the normal coordinates in Eq. $(\mathrm{C} 11)$ can then be reduced to a multidimensional integral over the vacancy energy fluctuations given by

$$
\begin{aligned}
\rho_{a i j}(\varepsilon)= & \int_{\ldots} \int \frac{\prod_{i} d \delta \varepsilon_{i}}{\sqrt{(2 \pi)^{N} \operatorname{det} C}} \exp \left(-\sum_{i, j} C_{i j}^{-1} \delta \varepsilon_{i} \delta \varepsilon_{j} / 2\right) \\
& \times\left\langle\psi_{a i}\left|\delta\left(\varepsilon \hat{I}_{\mathrm{a}}-\hat{H}_{\mathrm{a}}\left(\left\{\delta \varepsilon_{i}\right\}_{i}\right)\right)\right| \psi_{a j}\right\rangle,
\end{aligned}
$$

where

$$
\hat{H}_{\mathrm{a}}\left(\left\{\delta \varepsilon_{a i}\right\}_{i}\right)=\hat{H}_{\mathrm{a}}^{(0)}+\sum_{\lambda} \delta \varepsilon_{i}\left|\psi_{a i}\right\rangle\left\langle\psi_{a i}\right|
$$

and

$$
C_{i j}=\left\langle\delta \varepsilon_{a i} \delta \varepsilon_{a j}\right\rangle=\sum_{\lambda} \varepsilon_{a i \lambda}^{\prime} \varepsilon_{a j \lambda}^{\prime} \frac{\hbar}{2 \omega_{\lambda}}
$$

is the correlation function for vacancy on-site energy fluctuations at zero temperature. Here we have not attempted to calculate this correlation function in terms of the phonon modes, but considered instead the uncorrelated limit corresponding to

$$
C_{i j}=\left\langle\delta \varepsilon_{a}^{2}\right\rangle \delta_{i j},
$$

and the fully correlated limit corresponding to

$$
C_{i j}=\left\langle\delta \varepsilon_{a}^{2}\right\rangle \text {. }
$$

Here, $\left\langle\delta \varepsilon_{a}^{2}\right\rangle$ is obtained from the phonon broadening of a single VS.

For a single vacancy, the DOS in Eq. (C14) simply reduces to a Gaussian line shape,

$$
\rho_{a}(\varepsilon)=g\left(\varepsilon-\varepsilon_{a}^{(0)}\right)=\frac{1}{\sqrt{2 \pi} \sigma} \exp \left[-\frac{\left(\varepsilon-\varepsilon_{a}^{(0)}\right)^{2}}{2 \sigma^{2}}\right],
$$

and is simply given by the fluctuations of the resonance energy induced by zero-point motion of the phonons. Here, $\sigma^{2}=\left\langle\delta \varepsilon_{a}^{2}\right\rangle$, and the full width at half maximum (FWHM) of the broadening is then given by

$$
\sigma_{\mathrm{FWHM}}=2 \sqrt{2 \ln 2\left\langle\delta \varepsilon_{a}^{2}\right\rangle} .
$$

In the uncorrelated limit for $C_{i j}$, the multivariate Gaussian distribution of $\delta \varepsilon_{i}$ is independent over the different sites. Accordingly, the LDOS in Eq. (C14) is obtained from Eqs. (C7) and (C11) by inserting the eigenenergies $\varepsilon_{\kappa}\left(\left\{\delta \varepsilon_{i}\right\}_{i}\right)$ and the eigenstates,

$$
\left|\psi_{\kappa}\left(\left\{\delta \varepsilon_{i}\right\}_{i}\right)\right\rangle=\sum_{j} c_{\kappa j}\left(\left\{\delta \varepsilon_{i}\right\}_{i}\right)\left|\psi_{a j}\right\rangle,
$$

of $\hat{H}_{\mathrm{a}}\left(\left\{\delta \varepsilon_{i}\right\}_{i}\right)$ into Eq. $(\mathrm{C} 15)$ as

$$
\begin{aligned}
\rho\left(\varepsilon, \vec{r}_{t}\right) \approx & \int_{\ldots} \int \prod_{i} d \delta \varepsilon_{i} g\left(\delta \varepsilon_{i}\right)\left|\sum_{j} c_{\kappa j}\left(\left\{\delta \varepsilon_{i}\right\}_{i}\right)\left\langle\mathbf{r}_{0} \mid \psi_{a j}\right\rangle\right|^{2} \\
& \times \delta\left(\varepsilon-\varepsilon_{\kappa}\left(\left\{\delta \varepsilon_{i}\right\}_{i}\right)\right) .
\end{aligned}
$$

The total DOS or the level distribution, $\rho(\varepsilon)$, is obtained by an integration over $\mathbf{r}_{0}$ and is then given by

$$
\rho(\varepsilon) \approx \sum_{\kappa} \int_{\ldots} \int \prod_{i} d \delta \varepsilon_{i} g\left(\delta \varepsilon_{i}\right) \delta\left(\varepsilon-\varepsilon_{\kappa}\left(\left\{\varepsilon_{i}\right\}_{i}\right)\right) .
$$

The eigenstates and eigenenergies of $\hat{H}_{\mathrm{a}}\left(\left\{\delta \varepsilon_{i}\right\}_{i}\right)$ will depend nonlinearly on the vacancy energy fluctuations $\left\{\delta \varepsilon_{i}\right\}_{i}$. This dependence can be solved analytically for the divacancy, and the LDOS and the level distribution can then be obtained by a numerical quadrature as detailed below. The LDOS and the level distributions of longer chains can be efficiently generated by a Monte-Carlo sampling of the Gaussian distributions in the multidimensional integrals over $\delta \varepsilon_{i}$ and a diagonalization of the corresponding $\hat{H}_{a}\left(\left\{\delta \varepsilon_{i}\right\}_{i}\right)$ for each sampled $\left\{\delta \varepsilon_{i}\right\}_{i}$.

Before showing explicitly how the results for the level distribution of a divacancy were obtained for uncorrelated vacancy energy fluctuations from Eq. (C23), we will first 
show how the results in Figs. 4(a)-4(d) for the divacancy were obtained. The energy levels in Figs. 4(c) and 4(d) were obtained by diagonalizing $\hat{H}_{a}\left(\delta \varepsilon_{1}, \delta \varepsilon_{2}\right)$ for fixed $\delta \varepsilon_{1}$ and $\delta \varepsilon_{2}$. The eigenenergies of this two-state, eigenvalue problem are given by

$$
\tilde{\varepsilon}_{a, b}=\varepsilon_{a}^{(0)}+\delta \varepsilon_{s} \pm \sqrt{t^{2}+\delta \varepsilon_{a s}^{2}},
$$

where

$$
\begin{gathered}
\delta \varepsilon_{s}=\frac{1}{2}\left(\delta \varepsilon_{1}+\delta \varepsilon_{2}\right) \\
\delta \varepsilon_{a s}=\frac{1}{2}\left(\delta \varepsilon_{1}-\delta \varepsilon_{2}\right)
\end{gathered}
$$

are the symmetric and antisymmetric components of $\delta \varepsilon_{1}$ and $\delta \varepsilon_{2}$, respectively. For a symmetric fluctuation, $\delta \varepsilon_{1}=\delta \varepsilon_{2}$, the two divacancy states

$$
\tilde{\varepsilon}_{a, b}=\varepsilon_{a}^{(0)}+\delta \varepsilon_{s} \pm t,
$$

will shift rigidly with $\delta \varepsilon_{s}$ [Fig. 4(c)], whereas an antisymmetric fluctuation, $\delta \varepsilon_{1}=-\delta \varepsilon_{2}$, will shift them with respect to each other with a minimum splitting of $2 t$ [Fig. 4(d)] as

$$
\tilde{\varepsilon}_{a, b}=\varepsilon_{a}^{(0)} \pm \sqrt{t^{2}+\delta \varepsilon_{a s}^{2}} .
$$

The two adiabatic potential energy curves in Figs. 4(a) and 4(b) were obtained by first minimizing the potential energy of the phonons,

$$
V\left(\left\{q_{\lambda}\right\}_{\lambda}\right)=\sum_{\lambda} \frac{\omega_{\lambda}^{2} q_{\lambda}^{2}}{2},
$$

with respect to $q_{\lambda}$ under the constraint that $\delta \varepsilon_{1}$ and $\delta \varepsilon_{2}$ are both fixed. The resulting minimum value of this potential energy is then given by

$$
V_{\min }\left(\delta \varepsilon_{1}, \delta \varepsilon_{2}\right)=\sum_{i j} \frac{1}{2} K_{i j}^{-1} \delta \varepsilon_{i} \delta \varepsilon_{j},
$$

where

$$
K_{i j}=\sum_{\lambda} \frac{\varepsilon_{a i \lambda}^{\prime} \varepsilon_{a j \lambda}^{\prime}}{\omega_{\lambda}^{2}}
$$

Under the assumption that the phonon relaxation energy of a localized vacancy state in the divacancy, as obtained by minimizing, $V_{\min }\left(\delta \varepsilon_{1}, \delta \varepsilon_{2}\right)+\delta \varepsilon_{i}$, is the same as for the isolated single vacancy then $K_{12}=0$ and

$$
V_{\min }\left(\delta \varepsilon_{1}, \delta \varepsilon_{2}\right)=\sum_{i} \frac{\delta \varepsilon_{i}^{2}}{4 E^{\mathrm{rel}}},
$$

where

$$
E^{\mathrm{rel}}=\frac{K_{11}}{2}=\frac{K_{22}}{2}
$$

is the phonon relaxation energy for a single vacancy. The adiabatic potential energy curves $V_{a, b}$ for $\delta_{a s}$ and $\delta_{s}$ in Figs. 4(a) and 4(b) are then given by

$$
\begin{gathered}
V_{a, b}\left(\delta \varepsilon_{s}\right)=\varepsilon_{a}^{(0)}+\delta \varepsilon_{s} \pm t+\frac{\delta \varepsilon_{s}^{2}}{2 E^{\mathrm{rel}}}, \\
V_{a, b}\left(\delta \varepsilon_{a s}\right)=\varepsilon_{a}^{(0)} \pm \sqrt{t^{2}+\delta \varepsilon_{a s}^{2}}+\frac{\delta \varepsilon_{a s}^{2}}{2 E^{\mathrm{rel}}} .
\end{gathered}
$$

The results in Figs. 4(a)-4(d) were based on parameters appropriate for the $3 \mathrm{NN}$ vacancy $\varepsilon_{a}^{(0)}=2.73 \mathrm{eV}$ and $t=$ $0.13 \mathrm{eV}$. The relaxation energy was estimated using the following expression, as obtained from Eqs. (C16) and (C31):

$$
E^{\mathrm{rel}}=\frac{\left\langle\delta \varepsilon_{a}^{2}\right\rangle}{\hbar \bar{\omega}},
$$

where the characteristic phonon frequency $\bar{\omega}$ is defined in terms of a ratio of frequency moment as

$$
\bar{\omega}=\frac{\sum_{\lambda}\left(\epsilon_{a \lambda}^{\prime}\right)^{2} \omega_{\lambda}^{-1}}{\sum_{\lambda}\left(\epsilon_{a \lambda}^{\prime}\right)^{2} \omega_{\lambda}^{-2}} .
$$

Using the value $32 \mathrm{meV}$ of a typical $\mathrm{NaCl}$ optical phonon energy [40] for $\hbar \bar{\omega}$ and $\sqrt{\left\langle\delta \varepsilon_{a}^{2}\right\rangle}=0.115 \mathrm{eV}$ as obtained from the observed $\sigma_{\mathrm{FWHM}}=0.27 \mathrm{eV}$, this estimate gives $E^{\text {rel }}=0.41 \mathrm{eV}$. Note that in our previous work [32] on the phonon broadening of the single vacancies, the broadening was extracted using the calculated relaxation energy of about $1 \mathrm{eV}$, which using the same value for $\hbar \bar{\omega}$ gave a somewhat larger value for the phonon broadening than the observed one.

Now we proceed by showing the results for the level distribution of a divacancy. Using the result for the energy levels in Eq. (C24), the level distribution for uncorrelated vacancy energy fluctuations, Eq. (C23), can be expressed as

$$
\begin{aligned}
\rho(\varepsilon)= & \sum_{ \pm} \iint d \delta \varepsilon_{s} d \delta \varepsilon_{a s} \tilde{g}\left(\delta \varepsilon_{s}\right) \tilde{g}\left(\delta \varepsilon_{a s}\right) \\
& \times \delta\left(\varepsilon-\varepsilon_{a}^{(0)}-\delta \varepsilon_{s} \mp \sqrt{t^{2}+\delta \varepsilon_{a s}^{2}}\right),
\end{aligned}
$$

where $\tilde{g}(\delta \varepsilon)$ is the Gaussian function in Eq. (C19) but now with $\tilde{\sigma}^{2}=\sigma^{2} / 2=\left\langle\delta \varepsilon_{a}^{2}\right\rangle / 2$. The linear dependence of the divacancy energies on $\delta \varepsilon_{s}$ show that $\rho(\varepsilon)$ is simply a convolution of $\tilde{g}(\varepsilon)$ with the level distribution, $\rho_{a s}(\varepsilon)$ for antisymmetric fluctuations of the vacancy energies, which is given by

$$
\rho_{a s}(\varepsilon)=\sum_{ \pm} \int d \delta \varepsilon_{a s} \tilde{g}\left(\delta \varepsilon_{a s}\right) \delta\left(\varepsilon-\varepsilon_{a}^{(0)} \mp \sqrt{t^{2}+\delta \varepsilon_{a s}^{2}}\right) .
$$

The corresponding distribution for symmetric fluctuations of the vacancy energies is then given by

$$
\rho_{s}(\varepsilon)=\sum_{ \pm} \tilde{g}\left(\varepsilon-\varepsilon_{a}^{(0)} \mp t\right) .
$$

Finally, in the fully correlated limit for the chain of the vacancies, $\delta \varepsilon_{a i}=\delta \varepsilon_{a}$ and the probability distribution collapses to a single Gaussian distribution $g\left(\delta \varepsilon_{a}\right)$. In this case, $\tilde{H}_{e}\left(\left\{\varepsilon_{a i}\right\}_{i}\right)=\tilde{H}_{e}^{(0)}+\delta \varepsilon_{a} \tilde{I}_{a}$, and its eigenstates are the same as for $\tilde{H}_{e}^{(0)}$ and the eigenenergies $\varepsilon_{\kappa}$ of $\tilde{H}_{e}$ are simply shifted by $\delta \varepsilon_{a}$ from the eigenenergies $\varepsilon_{\kappa}^{(0)}$ of $\tilde{H}_{e}^{(0)}$. Thus, in this case, the LDOS and the level distribution are given by

$$
\rho^{c}\left(\varepsilon, \vec{r}_{t}\right) \approx \sum_{\kappa}\left|\sum_{i} c_{\kappa i}\left\langle\mathbf{r}_{0} \mid \psi_{a i}\right\rangle\right|^{2} g\left(\varepsilon-\varepsilon_{\kappa}^{(0)}\right)
$$

and

$$
\rho^{c}(\varepsilon) \approx \sum_{\kappa} g\left(\varepsilon-\varepsilon_{\kappa}^{(0)}\right)
$$



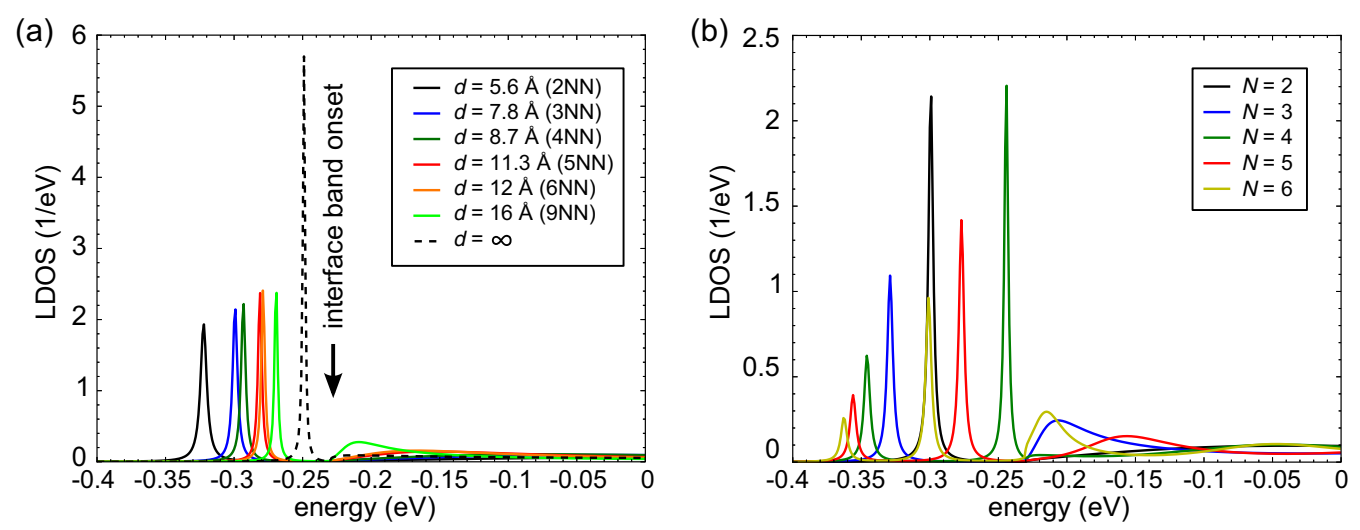

FIG. 7. (Color online) (a) LISs of $\mathrm{Cl}$ divacancies with different intervacancy distances. (b) LISs of small $\mathrm{Cl}$ vacancy chains with $N=2-6$ vacancies in the $5 \mathrm{NN}$ configuration. The peaks are broadened by adding a small imaginary part of $1 \mathrm{meV}$ to the energy.

This result corresponds simply to the same Gaussian broadening of all the vacancy levels $\varepsilon_{\kappa}^{(0)}$.

\section{Multiple scattering model for the formation of localized interface-states}

In the analysis of the formation of localized interfacestates from the interaction of the chains of vacancies with the interface-state band, we use a simple phenomenological, one-electron model [41]. The interface-state band is modeled by a $2 \mathrm{D}$ free-electron band with an effective mass $m^{*}$ and a band onset $\varepsilon_{B}$. The propagation of the electrons in the interface-state band is described by the one-electron Green function

$$
\begin{aligned}
\left\langle\vec{R}\left|\hat{g}_{0}(\varepsilon)\right| \vec{R}^{\prime}\right\rangle & =g_{0}\left(\left|\vec{R}-\vec{R}^{\prime}\right|, \varepsilon\right) \\
& =-\frac{i m^{*}}{2 \hbar^{2}} \mathrm{H}_{0}^{(1)}\left(K\left|\vec{R}-\vec{R}^{\prime}\right|\right),
\end{aligned}
$$

where $K=\sqrt{2 m^{*}\left(\varepsilon-\varepsilon_{B}\right)} / \hbar$ is the $2 \mathrm{D}$ wave vector and $\mathrm{H}_{0}^{(1)}(x)$ is the Hankel function of the first kind. The imaginary part of the diagonal part of the Green function gives the LDOS of the interface-state band, which has an onset at $\varepsilon_{B}$ and is constant over the interface state band.

The low-energy scattering of the interface-state electrons from a single vacancy located at $\vec{R}$ is described by a zerorange, $\sigma$-wave (zero angular momentum) scatterer defined by a transition matrix $t_{0}(\varepsilon)|\vec{R}\rangle\langle\vec{R}|$ containing only a single parameter $\varepsilon_{0}[41,42]$ :

$$
t_{0}(\varepsilon)=\frac{2 \pi \hbar^{2}}{m^{*}} \frac{1}{i \pi-\ln \left(\left(\varepsilon-\varepsilon_{B}\right) / \varepsilon_{0}\right)} .
$$

For an attractive interaction, $\varepsilon_{0}>0$, and $t_{0}(\varepsilon)$ has a simple pole at the energy $\varepsilon_{B}-\varepsilon_{0}$ corresponding to the bound state energy of the LIS of the single vacancy.

(a)

(b)

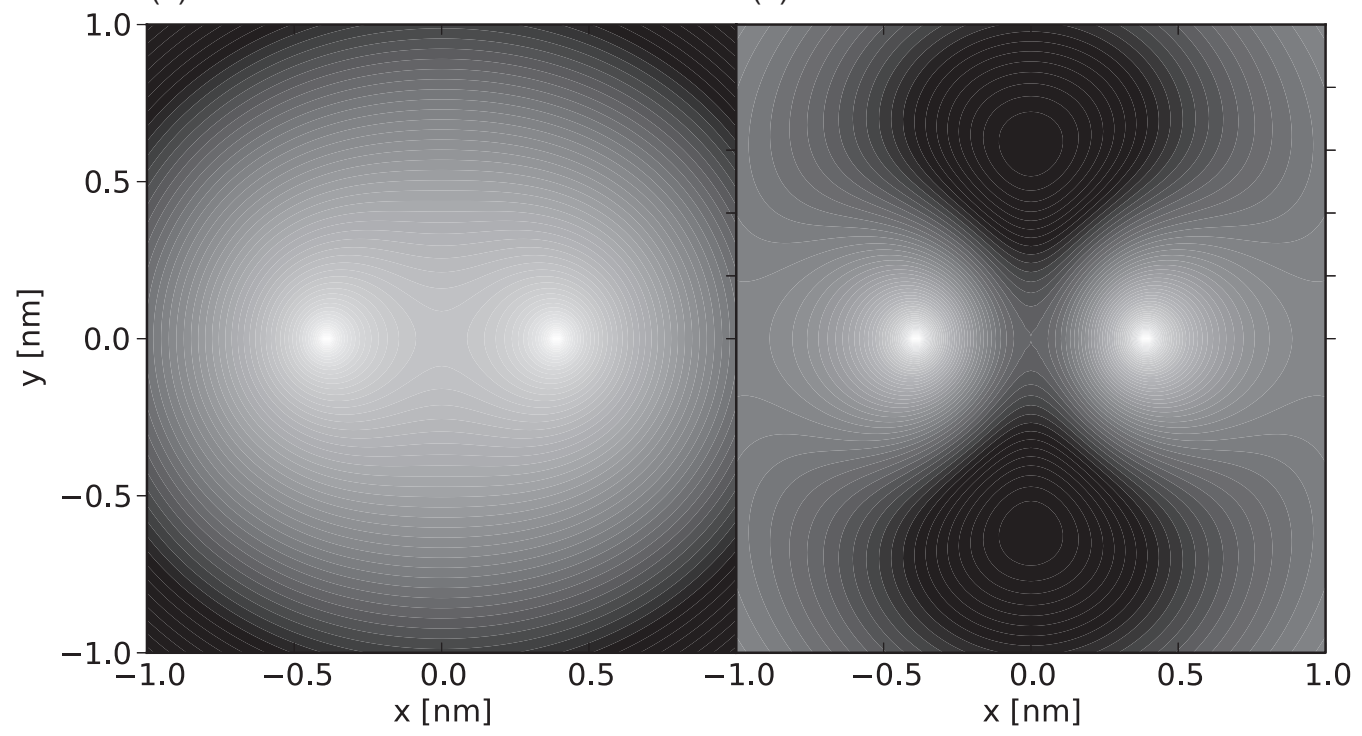

FIG. 8. Contour plots of the logarithm of the LDOS of (a) the LIS $(\varepsilon=-299 \mathrm{meV})$ and (b) interface states in the interface-state band $(\varepsilon=-130 \mathrm{meV})$ of the divacancy. 
The LDOS at the lateral position $\vec{R}_{t}$ of the tip apex from a linear chain of vacancies $i$ at positions $\vec{R}_{i}$ is obtained from solving the multiple scattering problem in similar manner as done in Ref. [43]. From the Dyson equation, one obtains

$\left\langle\vec{R}_{t}|\hat{g}(\varepsilon)| \vec{R}_{t}\right\rangle=\left\langle\vec{R}_{t}\left|\hat{g}_{0}(\varepsilon)\right| \vec{R}_{t}\right\rangle+\sum_{i}\left\langle\vec{R}_{t}\left|\hat{g}_{0}(\varepsilon)\right| \vec{R}_{i}\right\rangle a_{i}(\varepsilon),(\mathrm{C} 46)$

where the amplitude $a_{i}(\varepsilon)$ at a vacancy $i$ satisfies

$$
a_{i}(\varepsilon)=t_{0}(\varepsilon)\left\langle\vec{R}_{i}\left|\hat{g}_{0}(\varepsilon)\right| \vec{r}_{t}\right\rangle+t_{0}(\varepsilon) \sum_{j}\left\langle\vec{R}_{i}\left|\hat{g}_{0}(\varepsilon)\right| \vec{R}_{j}\right\rangle a_{j}(\varepsilon) .
$$

[1] N. Oncel, J. Phys.: Condens. Matter 20, 393001 (2008).

[2] N. Nilius, T. M. Wallis, and W. Ho, Science 297, 1853 (2002).

[3] S. Loth, S. Baumann, C. P. Lutz, D. M. Eigler, and A. J. Heinrich, Science 335, 196 (2012).

[4] C. Blumenstein, J. Schafer, S. Mietke, S. Meyer, A. Dollinger, M. Lochner, X. Y. Cui, L. Patthey, R. Matzdorf, and R. Claessen, Nature Phys. 7, 776 (2011).

[5] S. Nadj-Perge, I. K. Drozdov, J. Li, H. Chen, S. Jeon, J. Seo, A. H. MacDonald, B. A. Bernevig, and A. Yazdani, Science 346, 602 (2014).

[6] J. N. Crain and D. T. Pierce, Science 307, 703 (2005).

[7] I. Barke, R. Bennewitz, J. Crain, S. Erwin, A. Kirakosian, J. McChesney, and F. Himpsel, Solid State Commun. 142, 617 (2007).

[8] S. Fölsch, J. Yang, C. Nacci, and K. Kanisawa, Phys. Rev. Lett. 103, 096104 (2009).

[9] S. Wang, W. Wang, and N. Lin, Phys. Rev. Lett. 106, 206803 (2011).

[10] A. Riss, S. Wickenburg, P. Gorman, L. Z. Tan, H.-Z. Tsai, D. G. de Oteyza, Y.-C. Chen, A. J. Bradley, M. M. Ugeda, G. Etkin, S. G. Louie, F. R. Fischer, and M. F. Crommie, Nano Lett. 14, 2251 (2014).

[11] J. Repp, P. Liljeroth, and G. Meyer, Nat. Phys. 6, 975 (2010).

[12] F. E. Olsson, M. Persson, A. G. Borisov, J. P. Gauyacq, J. Lagoute, and S. Fölsch, Phys. Rev. Lett. 93, 206803 (2004).

[13] S. Fölsch, J. Martínez-Blanco, J. Yang, K. Kanisawa, and S. C. Erwin, Nat. Nanotechnol. 9, 505 (2014).

[14] P. M. Koenraad and M. E. Flatté, Nat. Mat. 10, 91 (2011).

[15] S. Schofield, P. Studer, C. Hirjibehedin, N. Curson, G. Aeppli, and D. Bowler, Nat. Commun. 4, 1649 (2013).

[16] A. Lorke, J. P. Kotthaus, and K. Ploog, Phys. Rev. Lett. 64, 2559 (1990).

[17] S. Hameau, Y. Guldner, O. Verzelen, R. Ferreira, G. Bastard, J. Zeman, A. Lemaître, and J. M. Gérard, Phys. Rev. Lett. 83, 4152 (1999).

[18] M. B. Haider, J. L. Pitters, G. A. DiLabio, L. Livadaru, J. Y. Mutus, and R. A. Wolkow, Phys. Rev. Lett. 102, 046805 (2009).

[19] Z. Sun, I. Swart, C. Delerue, D. Vanmaekelbergh, and P. Liljeroth, Phys. Rev. Lett. 102, 196401 (2009).

[20] R. Leturcq, C. Stampfer, K. Inderbitzin, L. Durrer, C. Hierold, E. Mariani, M. G. Schultz, F. von Oppen, and K. Ensslin, Nat. Phys. 5, 327 (2009).

[21] K. Seufert, W. Auwärter, F. J. Garcia de Abajo, D. Ecija, S. Vijayaraghavan, S. Joshi, and J. V. Barth, Nano Lett. 13, 6130 (2013).
This linear system of equations for the amplitudes $a_{i}(\varepsilon)$ is readily solved numerically, and the LDOS is obtained from $\left\langle\vec{R}_{0}|\hat{g}(\varepsilon)| \vec{R}_{0}\right\rangle$ in Eq. (C46) after inserting $a_{i}(\varepsilon)$ into Eq. (C46). Using the experimentally determined values for $m^{\star}=0.46 m_{e}$ and $\varepsilon_{B}=-230 \mathrm{meV}$ for the interface state band [31] and fitting the $\varepsilon_{B}-\varepsilon_{0}$ to the observed value of $-19 \mathrm{meV}$ for the LIS of a single vacancy, the resulting LDOS, for different divacancies and $5 \mathrm{NN}$ vacancy chains are depicted in Fig. 7. The corresponding LIS bound state energies for the divacancies are shown in Table I. The images of the logarithm of the LDOS of the LIS for the $3 \mathrm{NN}$ divacancy and of the IS at an energy of $100 \mathrm{meV}$ above the band bottom are shown in Fig. 8.

[22] S. E. Andresen, R. Brenner, C. J. Wellard, C. Yang, T. Hopf, C. C. Escott, R. G. Clark, A. S. Dzurak, D. N. Jamieson, and L. C. Hollenberg, Nano Lett. 7, 2000 (2007).

[23] C. A. Mujica-Martinez, P. Nalbach, and M. Thorwart, Phys. Rev. Lett. 111, 016802 (2013).

[24] C. S. Lent, P. D. Tougaw, W. Porod, and G. H. Bernstein, Nanotechnol. 4, 49 (1993).

[25] N. Nilius, T. M. Wallis, and W. Ho, Phys. Rev. Lett. 90, 046808 (2003).

[26] J. Repp, G. Meyer, S. M. Stojković, A. Gourdon, and C. Joachim, Phys. Rev. Lett. 94, 026803 (2005).

[27] N. Nilius, T. M. Wallis, M. Persson, and W. Ho, Phys. Rev. Lett. 90, 196103 (2003).

[28] T. Sonnleitner, I. Swart, N. Pavliček, A. Pöllmann, and J. Repp, Phys. Rev. Lett. 107, 186103 (2011).

[29] S. Kawai, A. S. Foster, F. F. Canova, H. Onodera, S.-i. Kitamura, and E. Meyer, Nat. Commun. 5, 4403 (2014).

[30] R. Bennewitz, V. Barwich, M. Bammerlin, C. Loppacher, M. Guggisberg, A. Baratoff, E. Meyer, and H.-J. Güntherodt, Surf. Sci. 438, 289 (1999).

[31] J. Repp, G. Meyer, and K.-H. Rieder, Phys. Rev. Lett. 92, 036803 (2004).

[32] J. Repp, G. Meyer, S. Paavilainen, F. E. Olsson, and M. Persson, Phys. Rev. Lett. 95, 225503 (2005).

[33] L. Gross, F. Mohn, N. Moll, P. Liljeroth, and G. Meyer, Science 325, 1110 (2009).

[34] F. Mohn, B. Schuler, L. Gross, and G. Meyer, Appl. Phys. Lett. 102, 073109 (2013).

[35] L. Gross, B. Schuler, F. Mohn, N. Moll, N. Pavliček, W. Steurer, I. Scivetti, K. Kotsis, M. Persson, and G. Meyer, Phys. Rev. B 90, 155455 (2014).

[36] V. Zielasek, T. Hildebrandt, and M. Henzler, Phys. Rev. B 62, 2912 (2000).

[37] B. Simon, Ann. Phys. 97, 279 (1976).

[38] J. Tersoff and D. R. Hamann, Phys. Rev. Lett. 50, 1998 (1983).

[39] J. Tersoff and D. R. Hamann, Phys. Rev. B 31, 805 (1985).

[40] J. D. Dow and D. Redfield, Phys. Rev. B 5, 594 (1972).

[41] P. Hyldgaard and M. Persson, J. Phys.: Cond. Matt. 12, L13 (2000).

[42] A. Farrell and B. van Zyl, Can. J. Phys. 88, 817 (2010).

[43] E. J. Heller, M. F. Crommie, C. P. Lutz, and D. M. Eigler, Nature (London) 369, 464 (1994). 